\title{
Numerical Study of Statistical Properties of the Galactic Center Distance Estimate from the Geometry of Spiral Arm Segments
}

\author{
I. I. Nikiforov* and A. V. Veselova \\ St. Petersburg State University, Universitetskii pr. 28, \\ Staryi Peterhof, St. Petersburg, 198504 Russia
}

\begin{abstract}
The influence of various factors on the statistical properties of the Galactic center distance $\left(R_{0}\right)$ estimate obtained by solving the general problem of determining the geometric parameters of a Galactic spiral arm from its segment with the inclusion of the distance to the spiral pole, i.e., $R_{0}$, in the set of parameters has been studied by the Monte Carlo method. Our numerical simulations have been performed for the model segments representing the Perseus and Scutum arms based on masers in high-mass star forming regions. We show that the uncertainty in the present-day parallax measurements for these objects systematically decreases (!) with increasing heliocentric distance, while the relative uncertainty in the parallaxes is, on average, approximately constant. This lucky circumstance increases significantly (by a factor of 1.4-1.7) the accuracy of estimating $R_{0}$ from the arm segment traced by masers. Our numerical experiments provide evidence for the consistency of the $R_{0}$ estimate from the spiral-segment geometry. The significant biases of the estimate detected only for the Scutum arm are caused mainly by the random parallax errors, the small angular extent of the segment, and the small number of objects representing it. The dispersion of the $R_{0}$ estimate depends most strongly on the angular extent of the segment and the parallax uncertainty if the latter, on average, does not depend on the distance. The remaining parameters, except for the pitch angle, exert an equally significant, but weaker influence on the statistical accuracy of the estimate. When the data on 3-8 segments are processed simultaneously, the predicted standard error of the final estimate is $\sigma_{R_{0}} \simeq 0.5-0.3 \mathrm{kpc}$, respectively. The accuracy can be improved by increasing the extent of the identified segments and the number of objects belonging to them. The method of determining $R_{0}$ from spiral segments has turned out to be operable for a wide set of possible parameters even when using an L-estimator (median). This makes the development of a more complex method based on an M-estimator, which allows one to properly take into account the measuring and natural dispersions of objects relative to the arm center line and, thus, to avoid the biases of the parameter estimates, meaningful.
\end{abstract}

Keywords: solar Galactocentric distance, spiral structure, maser sources, spatial distribution, Galaxy (Milky Way).

*E-mail: nii@astro.spbu.ru 


\section{INTRODUCTION}

Recent progress in the observational data has made it possible to distinguish features in the Galactic spiral structure that are sharp in the sense of a strong apparent concentration to their center lines and simultaneously extended (for more details, see Nikiforov and Veselova 2018; hereafter NV18). This, in turn, has stimulated works on the spatial modeling of separate spiral arm segments when abandoning traditional assumptions for studies of the Galactic spiral pattern, about the number of arms in the Galaxy and the equality of the pitch angles for different arms (see NV18 for a review as well as, e.g., the recent papers by Griv et al. (2017) and Krishnan et al. (2017) and references therein). In our previous paper (NV18) we proposed to abandon yet another traditional assumptionthe preadopted distance $R_{0}$ from the Sun to the pole of the spiral arms, i.e., the distance to the Galactic center. If in this problem $R_{0}$ is deemed a free parameter, along with the segment parameters, then this, on the one hand, allows the spiral segments to be modeled in a more general form, which takes into account, in particular, the noticeable correlation between $R_{0}$ and the pitch angle (shown in NV18), and, on the other hand, potentially opens a new approach to determining $R_{0}$ in the class of spatial methods (for a review, see Nikiforov 2004) - from the geometry of spiral arms.

A simplified (three-point) method of solving the problem was developed in NV18 to test this approach. Its application in the same paper to a sample of objects (masers with trigonometric parallaxes), on the whole, confirmed that the new approach is operable: from masers in two arms (Perseus and Scutum) we managed to obtain reliable results based on which we deduced an estimate of $R_{0}=8.8 \pm 0.5 \mathrm{kpc}$.

In this paper we use the three-point method to numerically study the statistical properties of the $R_{0}$ estimate from the geometry of a spiral arm as a function of problem parameters. The method consists in obtaining the solution for three segment parameters, including $R_{0}$, from the positions of its three representative points. Being relatively easy to implement, this simplified method allows a large number of numerical experiments to be performed in a reasonable time. The latter is important for achieving the main goal of this paper - to evaluate the applicability conditions and the capabilities of the new approach to finding $R_{0}$ both for present-day data and in prospect.

\section{MONTE CARLO SIMULATIONS OF THE $R_{0}$ MEASUREMENT FROM THE SPIRAL-ARM GEOMETRY}

\subsection{Model Segment Parameters}

In accordance with the parametrization introduced in NV18, we will describe the spiral arm segment by the following set of parameters: the distance from the coordinate origin, i.e., from the Sun, to the pole of the model logarithmic spiral $\left(R_{0}\right)$; its pitch angle $(i)$; the spiral position angle at the solar circle $R=R_{0}\left(\lambda_{0}\right)$, where $R$ is the distance from a point in the Galactic plane to the spiral pole; the boundaries of the spiral segment 
in Galactocentric longitude $\left(\lambda_{1}^{s}\right.$ and $\left.\lambda_{2}^{s}\right)$ that define the angular extent of the segment $\left(\Delta \lambda=\lambda_{2}^{s}-\lambda_{1}^{s}\right)$; the number of objects representing the segment $(N)$; the natural rootmean-square (rms) scatter of objects across the segment $\left(\sigma_{\mathrm{w}}\right)$; the absolute $\left(\sigma_{\varpi}\right)$ or relative $\left(\sigma_{\varpi} / \varpi\right)$ rms measurement error of the parallax $\varpi$. Here the longitudes $\lambda$ are measured in the Galactic plane from the sunward direction clockwise when viewed from the North Galactic Pole, i.e., in the direction of Galactic rotation.

The introduction of errors into the parallaxes as allowance for the uncertainty in the heliocentric distances $r$ corresponds here to the use of data on masers with trigonometric parallaxes, the most promising type of reference objects for applying the new method (NV18). The variant with the specification of corresponds to the assumption that the mean parallax uncertainty within the segment may be deemed approximately constant. However, our analysis of the data from Reid et al. (2014) (see Subsection 2.2) showed that the absolute uncertainty $\sigma_{\varpi}$ of the present-day parallax measurements for masers depends systematically on their distance (decreases with increasing $r$ !), i.e., it is generally not constant, on average, for the segment. On the other hand, we found that the relative parallax uncertainty changes more weakly within the limits of spiral segments revealed by masers. Therefore, the variant with the specification of $\sigma_{\varpi} / \varpi$ describes better the present-day data for these objects. Since this tendency for the parallax error may not be retained in future, we considered both these variants of parameterizing the distance uncertainty.

The immediate goal of our numerical simulations is to find the mean bias and dispersion of the $R_{0}$ estimate from the spiral-segment geometry as a function of significant problem parameters $\left(\Delta \lambda, \sigma_{\mathrm{w}}, \sigma_{\varpi}, \sigma_{\varpi} / \varpi, N, i\right)$.

\subsection{Uncertainty in the Trigonometric Parallaxes from Masers}

To choose the representations of the parallax measurement errors in our numerical experiments, we analyzed the data from two catalogues containing estimates of the parallaxes $\varpi$ and their uncertainties $\sigma_{\varpi}$ for objects including masers: the more homogeneous catalogue by Reid et al. (2014), which contains 103 high-mass star forming regions (HMSFRs), and the catalogue by Rastorguev et al. (2017, hereafter the Rs17 catalogue), which includes the catalogue by Reid et al. (2014) with the replacement of old data by new ones for two objects (hereafter the Rd14 catalogue), as well as the data on 38 additional maser sources, out of which (as our study of the literature showed) only 9 belong to the class of HMSFRs, while the remaining ones belong to 14 other types (a total of 141 objects). We use the Rd14 catalogue, because Reid et al. (2014) pointed out the membership of masers in a particular spiral arm, and to compare the characteristics of the Rd14 objects and the sources added in Rs17.

Table 1 gives the median absolute $\left(\operatorname{Me} \sigma_{\varpi}\right)$ and relative $\left(\operatorname{Me}\left(\sigma_{\varpi} / \varpi\right)\right)$ parallax uncertainties found by us separately for the arm segments that were identified by Reid et al. (2014) for the entire Rd14 sample and for the HMSFR and non-HMSFR masers of the Rs17 catalogue. Since we will also need to choose $\sigma_{\mathrm{w}}$, the NV18 estimates of the natural scatter of masers across the segments for the arms for which we managed to resolve this parameter are listed in Table 1. 
Table 1. Dispersion characteristics of the spatial positions of masers from the data of the catalogues by Reid et al. (2014) and Rastorguev et al. (2017)

\begin{tabular}{l|c|c|c|c}
\hline Arm/sample, catalogue & $N$ & $\mathrm{Me} \sigma_{\varpi}, \mathrm{mas}$ & $\mathrm{Me}\left(\sigma_{\varpi} / \varpi\right)$ & $\sigma_{\mathrm{w}}, \mathrm{kpc}$ \\
\hline Scutum (Sct), Rd14 & 17 & 0.028 & 0.100 & \\
Sagittarius (Sgr), Rd14 & 18 & 0.033 & 0.085 & $0.29 \pm 0.08$ \\
Local (Loc), Rd14 & 25 & 0.035 & 0.054 & $0.30 \pm 0.05$ \\
Perseus (Per), Rd14 & 24 & 0.018 & 0.061 & $0.35 \pm 0.05$ \\
Outer (Out), Rd14 & 6 & 0.012 & 0.070 & - \\
\hline All objects (All), Rd14 & 103 & 0.025 & 0.069 & - \\
HMSFR masers, Rs17 & 112 & 0.025 & 0.073 & - \\
Non-HMSFR masers, Rs17 & 29 & 0.037 & 0.063 & - \\
\hline
\end{tabular}

$N$ is the number of objects; $\operatorname{Me} \sigma_{\varpi}$ and $\operatorname{Me}\left(\sigma_{\varpi} / \varpi\right)$ are the median absolute and relative parallax errors; $\sigma_{\mathrm{w}}$ is the natural rms scatter of objects across the segment inferred in NV18 for the final estimate of $R_{0}=8.8 \mathrm{kpc}$.

The $\sigma_{\varpi}=$ const model for a segment corresponds to the experience of stellar parallax measurements. For example, the median of the standard parallax errors in the Hipparcos catalogue is virtually independent of the stellar magnitude and begins to increase only as the limiting magnitude of the catalogue is approached (Fig. 1 in Mignard 2000), implying that the mean parallax uncertainty is approximately constant as one recedes from the Sun and increases only at distances close to the limiting ones for the catalogue. However, for masers the behavior of the mean $\sigma_{\varpi}$ with distance turned out to be much better than the expected one by analogy with stars. Me $\sigma_{\varpi}$ in Table 1 decreases as the segment recedes from the Sun, taking the greatest value for the Local arm and the smallest ones for the Scutum arm (in the inner Galaxy) and the Outer arm (outside the solar circle). A direct comparison of the catalogued $\sigma_{\varpi}$ and $r=1 / \varpi$ (Figs. 1a and 2a) shows that the parallax uncertainty, on average, drops (!) with distance (approximately hyperbolically), with the mean $\sigma_{\varpi}$ levels at the boundaries of the $r$ interval represented by the data differing by several times for both catalogues.

Note that the HMSFR masers added in Rs17 hardly change the median parallax uncertainties for objects of this class (Table 1$)$ and are located on the $\left(r, \sigma_{\varpi}\right)$ plane in good agreement with the Rd14 masers (Fig. 2a). Me $\sigma_{\varpi}=0.037_{-0.007}^{+0.033}$ for the non-HMSFR masers is higher than $\mathrm{Me} \sigma_{\varpi}=0.025_{-0.003}^{+0.004}$ for the HMSFR masers from the Rs17 catalogue by a factor of 1.5. Although these medians formally differ insignificantly, the overwhelming majority of non-HMSFR sources (25 of 29) have $\sigma_{\varpi}$ equal to or larger than the median one for the HMSFR masers (Fig. 2a). The slightly smaller $\operatorname{Me}\left(\sigma_{\varpi} / \varpi\right)$ for the non-HMSFR sources than that for the HMSFR ones (Table 1) is unrepresentative, because the former are predominantly near the Sun (Fig. 2b). In what follows, we will rely on the results for the HMSFR masers forming more homogeneous samples with more accurate parallax 

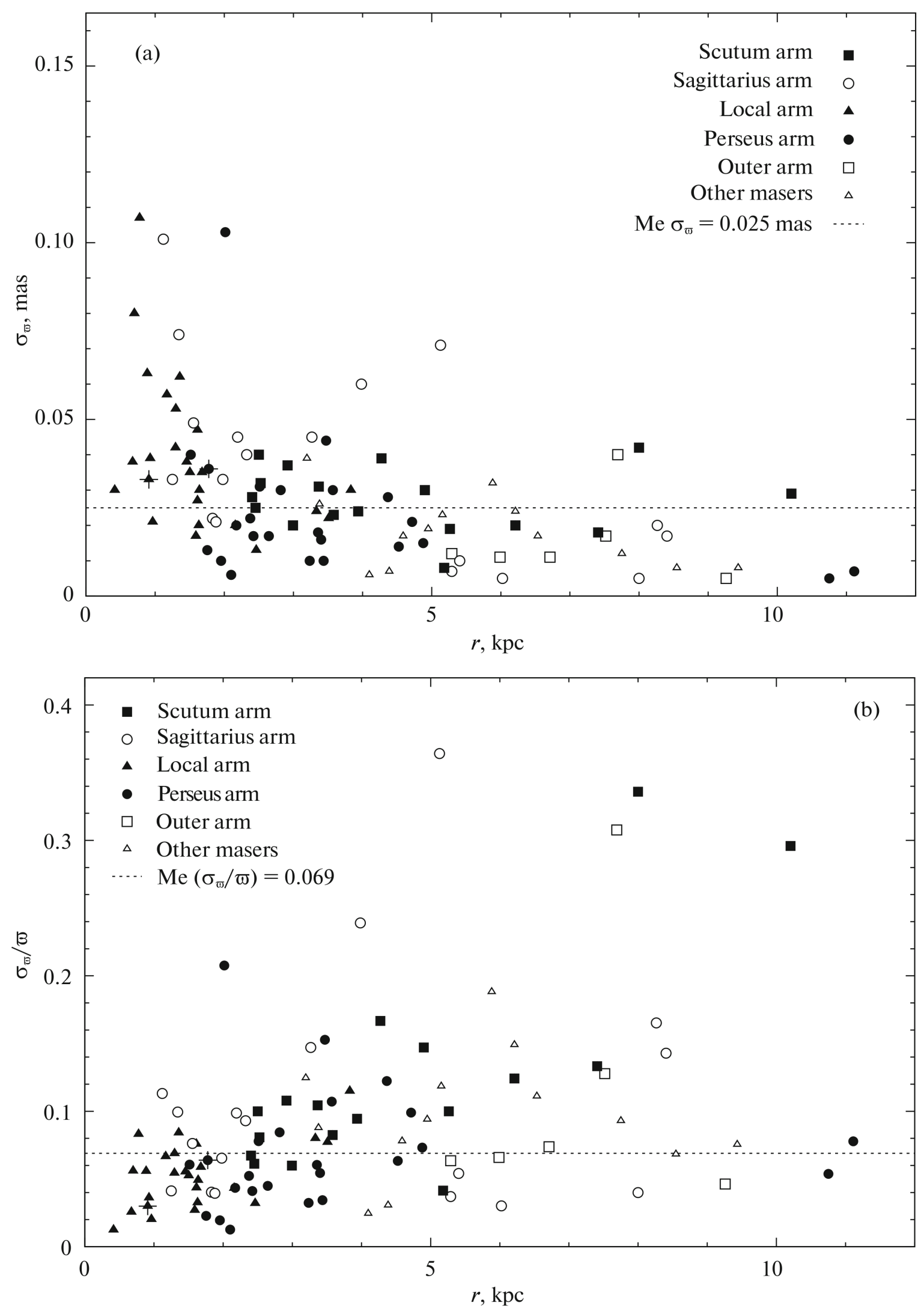

Fig. 1. Absolute (a) and relative (b) uncertainties of the parallax measurements versus heliocentric distance for the Rd14 masers. The crosses mark the two objects the data on which were replaced with the newer ones from the Rs17 catalogue. The dashed lines mark the median values of $\sigma_{\varpi}$ and $\sigma_{\varpi} / \varpi$ for the complete sample of masers in the catalogue. 

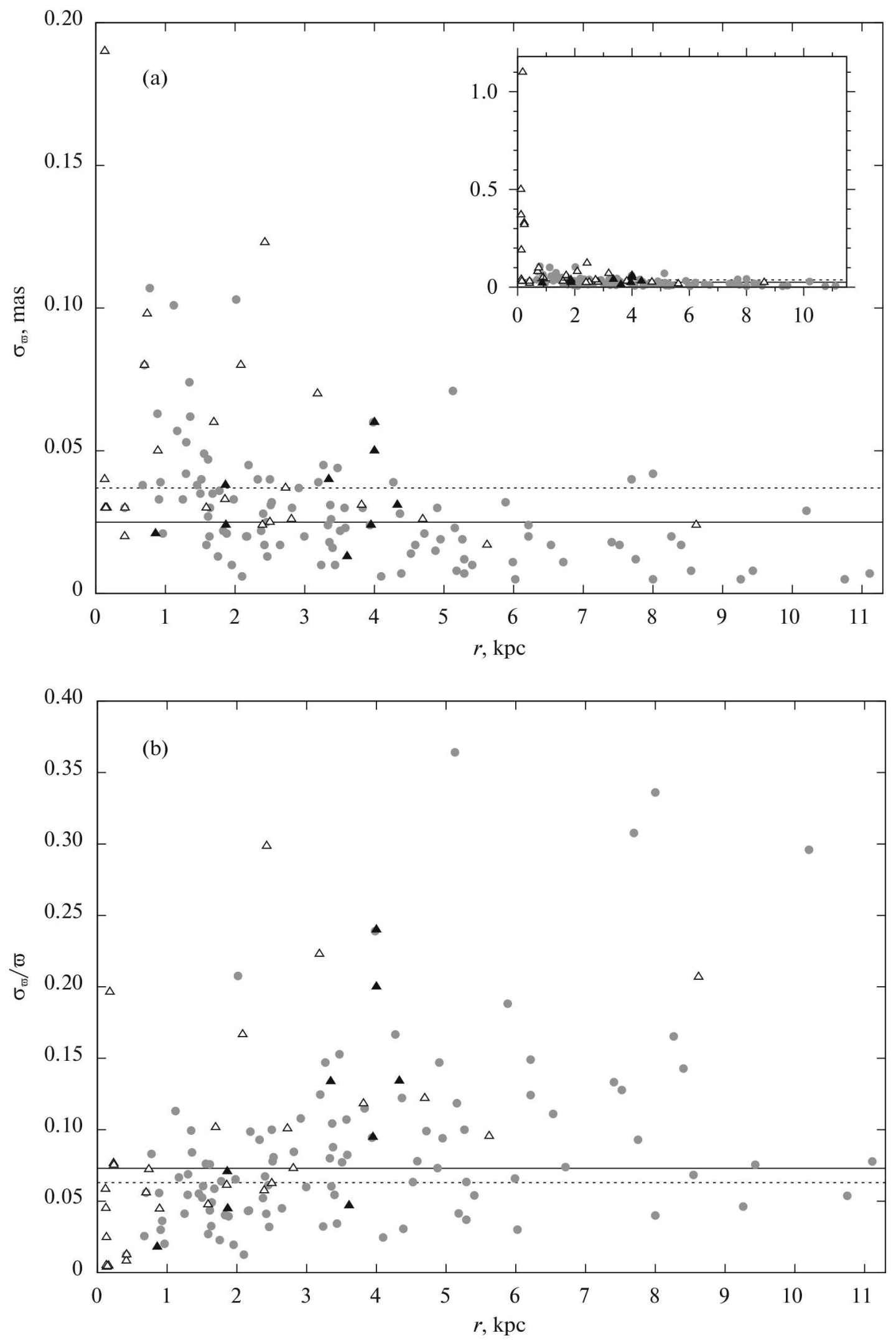

Fig. 2. Absolute (a) and relative (b) uncertainties of the parallax measurements versus heliocentric distance for the Rd14 masers (gray circles) and the additional Rs17 masers, HMSFRs (black triangles) and the remaining sources (white triangles). The solid and dashed lines mark the median values of $\sigma_{\varpi}$ and $\sigma_{\varpi} / \varpi$ for the complete sample of HMSFR masers in the Rs17 catalogue and for the remaining objects of the same catalogue, respectively. 
measurements.

To check the significance of these trends, we estimated the linear correlation coefficients $\kappa$ between the parallax uncertainties, on the one hand, and the distances or parallaxes, on the other hand, for each individual segment and for all HMSFR masers of the Rd14 and Rs17 catalogues (Table 2). For each estimate of the coefficient $\kappa\left(p_{1}, p_{2}\right)$ from the sample values of $N$ pairs of random variables $p_{1}$ and $p_{2}$ we calculated the probability $P_{\kappa}$ to obtain a correlation coefficient larger in absolute value than $\left|\kappa\left(p_{1}, p_{2}\right)\right|$ under the assumption that $p_{1}$ and $p_{2}$ were uncorrelated using the formula for small $N$

$$
P_{\kappa}=1-S_{N-2}(t), \quad t=\kappa\left(p_{1}, p_{2}\right) \sqrt{\frac{N-2}{1-\kappa^{2}\left(p_{1}, p_{2}\right)}},
$$

where $S_{N-2}(t)$ is Student's distribution with $N-2$ degrees of freedom (Press et al. 1997). $P_{\kappa}$ are also given in Table 2. The null hypothesis about the absence of a correlation may be rejected at $P_{\kappa}<0.05$. The values of $\kappa$ that are significant in accordance with this rule are highlighted in Table 2 in boldface.

The results presented in Table 2 show that the correlations between $\sigma_{\varpi}$ and $r$ (negative) and between $\sigma_{\varpi}$ and $\varpi$ (positive) are significant not only for the complete samples of HMSFRs, but also within the segments of the Local and Sagittarius arms separately. Since the tendency for $\sigma_{\varpi}$ to drop with $r$ is most pronounced at distances $r \lesssim 2.5 \mathrm{kpc}$ (Figs. 1a and 2a), it is less evident in the case of other segments, which occupy little this region (Perseus and Scutum arms) or do not pass through it at all (Outer arm) (Fig. 1a). However, when the two most distant masers of the Scutum arm segment are excluded, $\kappa\left(\sigma_{\varpi}, r\right)$ and $\kappa\left(\sigma_{\varpi}, \varpi\right)$ become significant for this feature as well. The closeness of the significant correlation coefficients to one another $\left(\kappa\left(\sigma_{\varpi}, r\right)=-0.55 \div-0.44, \kappa\left(\sigma_{\varpi}, \varpi\right)=+0.38 \div+0.60\right)$ suggests that in all these cases we are dealing with the same effects. In the opinion of Reid (2014), these may include: (1) the decrease in the apparent size of maser spots with distance, as a consequence of which the accuracy of astrometric measurements, other things being equal, increases; and (2) using a larger number of observations for some distant objects (e.g., W 49). In any case, the results obtained suggest that at the present epoch the accuracy of maser parallax measurements, on average, increases with distance and, consequently, the $\sigma_{\varpi}=$ const model for a segment is inconsistent with the present-day data. Note that the same tendency is independently traced by non-HMSFR masers, only with a shift toward larger $\sigma_{\varpi}$ (Fig. 2a).

Given the trend $\left\langle\sigma_{\varpi}\right\rangle \propto r^{-1}$, one might expect that the relative uncertainty $\sigma_{\varpi} / \varpi$, on average, does not change with $r$. A direct comparison of $\sigma_{\varpi} / \varpi$ with $r$ (Figs. 1b and 2b) shows that the scatter of $\sigma_{\varpi} / \varpi$ for the HMSFRs actually remains approximately constant at $r \lesssim 3.2 \mathrm{kpc}$. Outside this region, as $r$ increases, the values of $\sigma_{\varpi} / \varpi$ shift, on the whole, to large values and several measurements with a low relative accuracy appear. However, this tendency is much less pronounced than the reduction in $\left\langle\sigma_{\varpi}\right\rangle$ with $r$. Although for complete samples of HMSFRs the correlations between $\sigma_{\varpi} / \varpi$ and $r$ (positive) and between $\sigma_{\varpi} / \varpi$ and $\varpi$ (negative) turn out to be significant, for separate segments they are either insignificant or, for the Local and Scutum arms, are significant only formally (Table 2). 
Table 2. Linear correlation coefficients of the absolute and relative parallax uncertainties with the distance and parallax estimates for the HMSFR masers.

\begin{tabular}{l|cc|cc|cc|cc}
\hline $\begin{array}{l}\text { Arm/sample, } \\
\text { catalogue }\end{array}$ & $\kappa\left(\sigma_{\varpi}, r\right)$ & $P_{\kappa}$ & $\kappa\left(\sigma_{\varpi} / \varpi, r\right)$ & $P_{\kappa}$ & $\kappa\left(\sigma_{\varpi}, \varpi\right)$ & $P_{\kappa}$ & $\kappa\left(\sigma_{\varpi} / \varpi, \varpi\right)$ & $P_{\kappa}$ \\
\hline Sct, 14 & -0.10 & 0.69 & $+\mathbf{0 . 7 9}$ & 0.0003 & +0.23 & 0.38 & $\mathbf{- 0 . 6 5}$ & 0.0055 \\
Sct ${ }^{\mathrm{a}}, \mathrm{Rd} 14$ & $-\mathbf{0 . 5 5}$ & 0.035 & +0.46 & 0.076 & $+\mathbf{0 . 5 2}$ & 0.048 & -0.48 & 0.064 \\
Sgr, Rd14 & $-\mathbf{0 . 5 5}$ & 0.018 & +0.20 & 0.43 & $+\mathbf{0 . 6 0}$ & 0.0094 & -0.26 & 0.29 \\
Loc, Rd14 & $-\mathbf{0 . 4 7}$ & 0.020 & $+\mathbf{0 . 5 5}$ & 0.0047 & $+\mathbf{0 . 3 8}$ & 0.059 & $\mathbf{- 0 . 4 8}$ & 0.016 \\
Per, Rd14 & -0.33 & 0.11 & +0.08 & 0.72 & +0.35 & 0.10 & -0.18 & 0.41 \\
Out, Rd14 & +0.06 & 0.90 & +0.19 & 0.72 & -0.15 & 0.78 & -0.28 & 0.60 \\
\hline All, Rd14 & $\mathbf{- 0 . 4 7}$ & $8 \cdot 10^{-5}$ & $+\mathbf{0 . 4 1}$ & $3 \cdot 10^{-5}$ & $+\mathbf{0 . 4 8}$ & $2 \cdot 10^{-7}$ & $\mathbf{- 0 . 3 6}$ & 0.0002 \\
HMSFRs, Rs17 & $\mathbf{- 0 . 4 4}$ & 0.0001 & $+\mathbf{0 . 4 0}$ & 0.0001 & $\mathbf{+ 0 . 4 9}$ & 0.0002 & $\mathbf{- 0 . 3 5}$ & 0.0003 \\
\hline
\end{tabular}

${ }^{a}$ Without two masers of the Scutum arm at $r \geqslant 8 \mathrm{kpc}$.

$P_{\kappa} \equiv P\left(|\varkappa|>\left|\kappa\left(p_{1}, p_{2}\right)\right|\right)$ is the probability to obtain a correlation coefficient $\varkappa$ larger in absolute value than the measured $\left|\kappa\left(p_{1}, p_{2}\right)\right|$ in the absence of a correlation between the random variables $p_{1}$ and $p_{2}$. The significant correlation coefficients $\left(P_{\kappa}<0.05\right)$ are highlighted in boldface.

For example, for the Scutum arm the significance of the correlations is based only on the two most distant $(r \geqslant 8 \mathrm{kpc})$ objects that differ sharply from other masers of the same segment by large $\sigma_{\varpi} / \varpi$, which exceed $\operatorname{Me}\left(\sigma_{\varpi} / \varpi\right)$ both for the objects of the Scutum arm and for the entire Rd14 catalogue by several times (Fig. 1b, Table 1). When these two (of 17) objects are excluded, the null hypothesis is not rejected for the Scutum arm either (Table 2). For the Local arm the formal significance appeared only after the replacement of the data for one (!) object (L 1206) in Rs17. (No such situation arises in the case of correlations for $\sigma_{\varpi}$.) The relative scatter of $\operatorname{Me}\left(\sigma_{\varpi} / \varpi\right)$ for different segments is noticeably smaller than that of $\operatorname{Me}\left(\sigma_{\varpi}\right)$ (Table 1 ). Thus, although the correlation between $\sigma_{\varpi} / \varpi$ and $r$ apparently exists, it manifests itself weakly within the arm segments considered separately. Therefore, the $\sigma_{\varpi} / \varpi=$ const model for a separate segment may be taken as consistent, to a first approximation, with the present-day data on masers.

Since one of the probable causes of the reduction in $\left\langle\sigma_{\varpi}\right\rangle$ with $r$ (using a larger number of observations for distant masers) will not be necessarily retained in future, in our numerical simulations we consider both variants of allowance for the parallax uncertainty. Out of them, as will be shown below, the $\sigma_{\varpi} / \varpi=$ const model describes a more favorable situation for estimating $R_{0}$ from the spiral-segment geometry, while the $\sigma_{\varpi}=$ const model describes a less favorable situation. From the presented results and reasoning one might expect the behavior of $\left\langle\sigma_{\varpi}\right\rangle$ with $r$, most likely, to remain intermediate between these two cases in any further parallax measurements. 


\subsection{Modeling Procedure}

Consider three representative points $M_{1}, M_{2}$, and $M_{3}$ belonging to the segment of a model spiral with parameters $R_{0}, i, \lambda_{0}, \lambda_{1}^{\mathrm{s}}$, and $\Delta \lambda$ (see Subsection 2.1). Let us choose the positions of the points so that the adjacent points are equidistant from one another in Galactocentric longitude $\lambda$. The longitudes of points $M_{j}$ are then defined by the expression $\lambda_{j}=\lambda_{1}^{\mathrm{s}}+\Delta \lambda(2 j-1) / 6, j=1,2,3$. Thus, the distance between the adjacent points is $\Delta \lambda / 3$, the distance from the extreme point $\left(M_{1}\right.$ or $\left.M_{3}\right)$ to the nearest (in longitude) segment boundary is $\Delta \lambda / 6$. Each of points $M_{j}$ represents the position of one third of the segment in longitude. The equidistant configuration of the representative points corresponds to a uniform distribution of objects in $\lambda$, i.e., approximately uniform along the segment. This model describes satisfactorily the reality in many cases (see, e.g., the figures in Popova and Loktin (2005) and Dambis et al. (2015)). Therefore (and in order not to increase the number of problem parameters), here we will restrict ourselves only to this type of configurations.

To take into account the scatter of objects across the arm segment and the parallax uncertainty in our numerical experiments, the positions of the representative points $M_{j}$, $j=1,2,3$, of the model spiral were varied as follows. Each point $M_{j}$ was shifted randomly by distance $\rho_{j}$ in one or the other direction along the straight line perpendicular to the model spiral at point $M_{j}$; in this way we found point $M_{j}^{\prime}$ with parallax $\varpi_{j}^{\prime}$ (Fig. 3). Then, point $M_{j}^{\prime}$ was shifted along the line of sight $S M_{j}^{\prime}$ also randomly, which determined a pseudo-random point $\left(M_{j}^{\prime \prime}\right.$ in Fig. 3$)$ with parallax $\varpi_{j}^{\prime \prime}$.

Once the set of points $M_{1}^{\prime \prime}, M_{2}^{\prime \prime}$, and $M_{3}^{\prime \prime}$ had been obtained, we searched for the parameters of the spirals passing through them in one turn. In this case (i.e., within the three-point method), the values of $R_{0}$ for such spirals are found from the following equation (see NV18):

$$
\left(\Lambda_{3}-\Lambda_{2}\right) \ln R_{1}+\left(\Lambda_{1}-\Lambda_{3}\right) \ln R_{2}+\left(\Lambda_{2}-\Lambda_{1}\right) \ln R_{3}=0,
$$

where the Galactocentric distances $R_{j}$ of points $M_{j}^{\prime \prime}$ are expressed via the Cartesian heliocentric coordinates $X_{j}$ and $Y_{j}$ of these points from the formula

$$
R_{j}=\sqrt{R_{0}^{2}+X_{j}^{2}+Y_{j}^{2}-2 R_{0} X_{j}}, \quad j=1,2,3,
$$

while the nominal Galactocentric longitudes $\Lambda_{j}$ of points $M_{j}^{\prime \prime}\left(-\pi \leqslant \Lambda_{j}<\pi\right.$, see NV18) are defined by the formulas

$$
\sin \Lambda_{j}=\frac{Y_{j}}{R_{j}}, \quad \cos \Lambda_{j}=\frac{R_{0}-X_{j}}{R_{j}}, \quad j=1,2,3 .
$$

As shown in NV18, for the triplet of points taken from the spiral, i.e., for $M_{1}, M_{2}$, and $M_{3}$, Eq. (2) can have one or two additional roots. Therefore, for the triplet of pseudorandom points $M_{1}^{\prime \prime}, M_{2}^{\prime \prime}$, and $M_{3}^{\prime \prime}$ we can also obtain more than one (up to three) roots of Eq. (2). In such cases, among the values of $R_{0}$ found for a given triple we chose $R_{0}$ closest to its initial value for the model spiral and it was considered to be the solution for the 


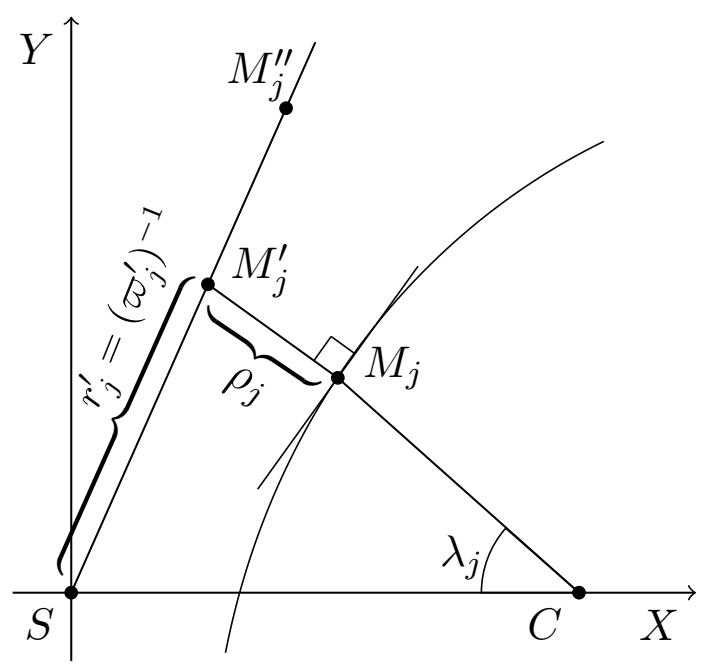

Fig. 3. Varying the position of a representative point $M_{j}$ along the perpendicular to the model spiral (point $M_{j}^{\prime}$ ) and along the line of sight (point $M_{j}^{\prime \prime}$ ). $S$ is the Sun's position, $C$ is the pole of the model spiral (Galactic center), and $(X, Y)$ is the Galactic plane.

triplet. In addition, as our numerical experiments showed, for points $M_{1}^{\prime \prime}, M_{2}^{\prime \prime}$, and $M_{3}^{\prime \prime}$ Eq. (2) may not have any roots. This means that the turn of at least one spiral cannot necessarily be drawn through three arbitrary points if the straight line on which the spiral pole can be located is specified. Such triplets were excluded from the subsequent analysis.

The value of $R_{0}$ found for the triplet $M_{1}^{\prime \prime}, M_{2}^{\prime \prime}$, and $M_{3}^{\prime \prime}$ also uniquely defines two other parameters $\left(i\right.$ and $\lambda_{0}$ ) of the spiral (Eqs. (12) and (13) in NV18). However, at this stage we do not consider the properties of the $i$ and $\lambda_{0}$ estimates as derived characteristics strongly correlating with $R_{0}$ (see NV18).

For the specified positions of the representative points $M_{j}$ and the chosen values of other parameters, we produced a set of $N_{\mathrm{MC}}$ triplets of pseudo-random points $\left\{M_{1, m}^{\prime \prime}, M_{2, m}^{\prime \prime}, M_{3, m}^{\prime \prime}\right\}_{m=1}^{N_{\mathrm{MC}}}$, where $N_{\mathrm{MC}}$ was taken in such a way that the number $N_{\text {sol }}$ of solutions for the problem of determining $R_{0}$, i.e., the number of triplets with a nonempty set of roots of Eq. (2). was 10 000. We assumed that $N$ objects with parallax estimates having an rms uncertainty $\sigma_{\varpi}$ or a relative uncertainty $\sigma_{\varpi} / \varpi$ were observed in the spiral segment with a standard scatter of object across the arm $\sigma_{\mathrm{w}}$. Each point $M_{j}$ models the mean position of one third of the segment. Therefore, the standard error in the position of $M_{j}$ in a direction across the arm may be approximately taken to be $\sigma_{\mathrm{w}} / \sqrt{N / 3}$, while the standard error in the parallax of the shifted point $M_{j}^{\prime}$ (see Fig. 3) may be taken to be $\sigma_{\varpi}\left(M_{j}^{\prime}\right) / \sqrt{N / 3}$, where $\sigma_{\varpi}\left(M_{j}^{\prime}\right)$ is the parallax uncertainty at point $M_{j}^{\prime}$. Hence, for the generation of points $M_{j, m}^{\prime \prime}, j=1,2,3, m=1,2, \ldots, N_{\mathrm{MC}}$, we varied $\rho_{j, m}$ (the shift of $M_{j, m}^{\prime}$ relative to $M_{j, m}$ ) according to a normal law with zero mean and standard deviation $\sigma_{\mathrm{w}} / \sqrt{N / 3}$ and $\varpi_{j, m}^{\prime \prime}$ according to a normal law with mean $\varpi_{j, m}^{\prime}$ and standard deviation $\sigma_{\varpi} / \sqrt{N / 3}$ in the case of a constant absolute parallax error for the segment or $\varpi_{j, m}^{\prime}\left(\sigma_{\varpi} / \varpi\right) / \sqrt{N / 3}$ in the case of a constant relative parallax error.

For the set of all our solutions $\left\{R_{0, s}\right\}, s=1,2, \ldots, N_{\text {sol }}$, we calculated the median Me $R_{0}$, the interval [Me $R_{0}-\sigma_{R_{0}}^{-}$, Me $R_{0}+\sigma_{R_{0}}^{+}$, with the probability for the individual 
$R_{0, s}$ estimate to fall into the latter being $\approx 68.3 \%$ ( $1 \sigma$ level $)$, and the $1 \sigma$ confidence interval [Me $R_{0}-\sigma^{-}\left(\mathrm{Me} R_{0}\right)$, Me $\left.R_{0}+\sigma^{-}\left(\mathrm{Me} R_{0}\right)\right]$ for the median. We determined the quantities $\sigma_{R_{0}}^{-}, \sigma_{R_{0}}^{+}$and $\sigma^{-}\left(\mathrm{Me} R_{0}\right), \sigma^{+}\left(\mathrm{Me} R_{0}\right)$ based on order statistics for the ordered set of $R_{0, s}$ estimates (see, e.g., Kobzar' 2006). We also calculated the mean bias of the $R_{0, s}$ estimates at given problem parameters: $\Delta R_{0}=\operatorname{Me} R_{0}-R_{0}$ with the confidence interval $\left[\Delta R_{0}-\sigma^{-}\left(\mathrm{Me} R_{0}\right), \Delta R_{0}+\sigma^{-}\left(\mathrm{Me} R_{0}\right)\right]$, where $R_{0}$ is the parameter of the original model spiral.

\subsection{Choosing the Basic Sets of Model Segment Parameters}

Our numerical simulations were performed for two families of model segments that represented the Perseus and Scutum arms, respectively. These cases approximately encompass the spread in probable configurations of segments revealed at the present epoch by objects with accurate heliocentric distances: in the local Galactocentric sector (containing the Sun) the Perseus arm passes outside the solar circle, i.e., far from the Galactic center, while the Scutum arm pases inside the circle $R=R_{0}$ relatively close to the center (see Fig. 13 in NV18 and Fig. 4 in this paper). In addition, the $R_{0}$ determination by the three-point method in NV18 turned out to be reliable precisely from masers in the Perseus and Scutum arms. Therefore, it now seems most interesting to investigate, also in prospect, the properties of the $R_{0}$ estimate from the geometry of these arm segments.

The parameter $R_{0}$ for the model segments was taken to be $8 \mathrm{kpc}$, in agreement with the recent mean ("best") values of $\left\langle R_{0}\right\rangle_{\text {best }}=(7.9 \div 8.3) \pm(0.1 \div 0.4) \mathrm{kpc}$ (Nikiforov and Smirnova 2013; Bland-Hawthorn and Gerhard 2016; de Grijs and Bono 2016; Camarillo et al. 2018) and the recent individual $R_{0}$ estimates (see, e.g., Rastorguev et al. 2017; Chen et al. 2018; Majaess et al. 2018). The initial $R_{0}$ in this problem is not an important parameter and did not change in our numerical experiments. For other parameters of the model segments of each of the two families we chose a set of such values that exactly or approximately corresponded to the characteristics found from the data of the catalogue by Reid et al. (2014) for masers in the Perseus and Scutum arms. We will call these sets and the values of the parameters themselves in them basic. These values are given in Table 3; the basic segments that correspond to them are shown in Fig. 4. Other sets of parameters for each family of models were formed by varying one of the parameters, while the remaining parameters retained their basic values.

The basic longitudes of the segment boundaries $\lambda_{1}^{\mathrm{s}}$ and $\lambda_{2}^{\mathrm{s}}$ correspond to the boundaries of the region occupied by the maser sources of a given arm. The basic values of $i_{0}$ and $\lambda_{0}$ were chosen so that the model segment passed through the region represented by the arm masers.

For the Perseus arm the basic scatter across the segment $\sigma_{\mathrm{w}}$ was taken to be $0.35 \mathrm{kpc}$, in accordance with the estimate that we obtained by applying the three-point method of determining the model spiral parameters to the segment masers (NV18). For the Scutum $\operatorname{arm} \sigma_{\mathrm{w}}=0.17 \mathrm{kpc}$ found by Reid et al. (2014) was taken for the same parameter. 


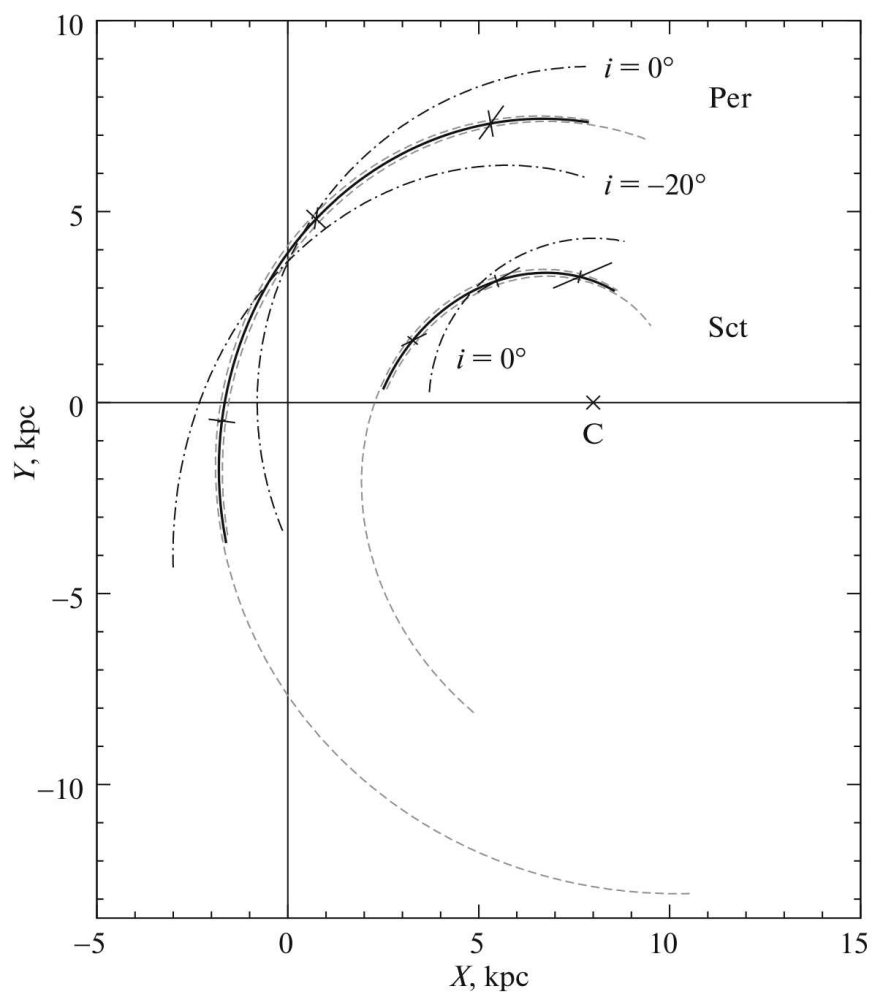

Fig. 4. Scheme of the model spiral segments considered in our numerical experiments: the basic segments (black curves), the segments of the largest angular extent $\Delta \lambda$ when fixing the boundary $\lambda_{1}^{\mathrm{s}}$ or $\lambda_{2}^{\mathrm{s}}$ (gray dashed curves, given with a slight shift in radius), and the segments with the largest and smallest pitch angles $i$ (black dash-dotted curves). The bars indicate the $1 \sigma$ distance uncertainty (at $\sigma_{\varpi} / \varpi=$ const) and the scatter across the arm at three representative points $M_{j}$ of the basic segments. For point $M_{1}$ of the Perseus arm the first of these bars exceeds only slightly the thickness of the model segment line. Point $C$ is the pole of all segments (Galactic center), the Sun is at the coordinate origin.

The basic values of $\sigma_{\varpi}$ and $\sigma_{\varpi} / \varpi$ were chosen to be equal to the medians Me $\sigma_{\varpi}$ and $\operatorname{Me}\left(\sigma_{\varpi} / \varpi\right)$ calculated for the masers of the corresponding arm (Table 1).

The basic number $N$ of objects representing the segment for the Perseus arm was taken to be equal to the number of masers in the catalogue by Reid et al. (2014) attributed to this arm. For the Scutum arm this number, for convenience (because each representative point $M_{j}$ represents $N / 3$ segment objects), was taken to be 18 , the number that is closest to the number of arm masers $(N=17)$ in the same catalogue and, at the same time, a multiple of three (Table 1).

Note that the basic set of parameters for the Perseus arm defines the segment crossing the Galactic center-anticenter line (Table 3, Fig. 4). In this case, in most of the generated triplets of representative points $M_{1, m}^{\prime \prime}, M_{2, m}^{\prime \prime}$, and $M_{3, m}^{\prime \prime}$ the individual points are located on different sides of this line. For such a configuration, in the overwhelming majority of cases, only one spiral passes through the triplet of points (see NV18); therefore, there is no need to choose the solution for $R_{0}$ from the set of roots of Eq. (2). The basic set for the Scutum arm specifies the segment that is completely on one side of the center-anticenter line, in quadrant I (Table 3, Fig. 4). In most cases, the generated triplets also turn out to 
Table 3. Basic sets of parameters for the model spiral segments representing the Perseus and Scutum arms in our numerical experiments

\begin{tabular}{lrr||lll}
\hline Parameter & Per & Sct & Parameter & Per & Sct \\
\hline$R_{0}, \mathrm{kpc}$ & 8.0 & 8.0 & $\sigma_{\varpi}$, mas & 0.018 & 0.028 \\
$i$ & $-10^{\circ}$ & $-20^{\circ}$ & $\sigma_{\varpi} / \varpi$ & 0.06 & 0.10 \\
$\lambda_{0}$ & $+61^{\circ}$ & $-55^{\circ}$ & $\sigma_{\mathrm{w}}, \mathrm{kpc}$ & 0.35 & 0.17 \\
\hline$\lambda_{1}^{\mathrm{s}}$ & $-21^{\circ}$ & $+3^{\circ}$ & & & \\
$\lambda_{2}^{\mathrm{s}}$ & $+88^{\circ}$ & $+100^{\circ}$ & $N$ & 24 & \multirow{2}{*}{$N$} \\
$\Delta \lambda$ & $109^{\circ}$ & $97^{\circ}$ & & & \\
\hline
\end{tabular}

be there. Two spirals always pass through the triplets arranged in this way for a nonempty set of roots of Eq. (2) and in nondegenerate cases (NV18). Therefore, choosing the root closest to the initial $R_{0}$ of the model spiral from the roots of (2) is necessary. The cases where there are no roots for some generated triplets (no solution for $R_{0}$ ) take place only for the sets of parameters at which the dispersion of points $M_{1, m}^{\prime \prime}, M_{2, m}^{\prime \prime}$, and $M_{3, m}^{\prime \prime}$ relative to the model segment is significant compared to the linear size and curvature radius of the segment. For example, for the family of Perseus arm models this occurs, very rarely, only at $\Delta \lambda=50^{\circ}$, a value that is less than half the basic one. For the family of the Scutum arm, on the contrary, such cases are commonplace, because the model segments of the family have considerably smaller linear extent and curvature radius than those of the Perseus arm segments (Fig. 4).

\section{RESULTS}

The results of our numerical simulations, the $1 \sigma$ statistical uncertainty $\left(\sigma_{R_{0}}^{+}, \sigma_{R_{0}}^{-}\right)$and the mean bias $\left(\Delta R_{0}\right)$ of the $R_{0}$ estimate from the spiral-segment geometry, for the basic sets of parameters (Table 3) and when replacing the basic value of one of the dispersions with zero in these sets are presented in Table 4. For the Perseus arm no significant biases $\Delta R_{0}$ were detected in all these variants of experiments. With the basic sets for the Scutum arm $\Delta R_{0}$ differ significantly from zero, substantially for the $\sigma_{\varpi}=$ const model. Comparison with the results for the same arm when one of the dispersions is set equal to zero (Table 4) shows that the bias is attributable to the parallax uncertainty (at $\sigma_{\varpi}=0$ mas, $\Delta R_{0}=0 \mathrm{kpc}$ within the error limits), but, on the other hand, the scatter across the arm, in addition to the nonzero $\sigma_{\varpi}$, increases $\Delta R_{0}$. The uncertainty in $R_{0}$ and the contribution of the parallax uncertainty to it depend strongly on the model for the latter within the arm: at $\sigma_{\varpi}=$ const the values of $\sigma_{R_{0}}^{ \pm}$are larger than those at $\sigma_{\varpi} / \varpi=$ const by $40-70 \%$. In the former case, the contribution of $\sigma_{\varpi}$ to the total uncertainty in $R_{0}$ clearly dominates (cf. the rows for $\sigma_{\varpi}=$ const $\neq 0$ and $\sigma_{\varpi}=0$ in Table 4); in the latter case, the contributions of both dispersions are, on the whole, comparable, while for the Perseus arm the scatter across the arm is even a more important factor (cf. the rows for $\sigma_{\varpi} / \varpi=$ const $\neq 0$ and $\sigma_{\varpi}=0$ in Table 4). For the two arms considered the uncertainties in $R_{0}$ with the basic sets of parameters turned out to be close in the case of $\sigma_{\varpi} / \varpi=$ const; the uncertainty in $R_{0}$ for the Scutum arm is slightly higher in the case of $\sigma_{\varpi}=$ const. 
Table 4. Statistical uncertainty $\left(\sigma_{R_{0}}^{+}, \sigma_{R_{0}}^{-}\right)$and mean bias $\left(\Delta R_{0}\right)$ of the $R_{0}$ estimate from the spiral-segment geometry for the basic set of the parameters for the Perseus and Scutum arm models and with one of the dispersions set equal to zero.

\begin{tabular}{|c|c|c|c|c|c|c|c|}
\hline \multicolumn{4}{|c|}{ Perseus arm } & \multicolumn{4}{|c|}{ Scutum arm } \\
\hline $\begin{array}{l}\sigma_{\varpi} / \varpi \\
\text { or } \sigma_{\varpi}, \text { mas }\end{array}$ & $\begin{array}{l}\sigma_{\mathrm{w}} \\
\mathrm{kpc}\end{array}$ & $\begin{array}{c}\sigma_{R_{0}}^{ \pm} \\
\mathrm{kpc}\end{array}$ & $\Delta R_{0}, \mathrm{kpc}$ & $\begin{array}{l}\sigma_{\varpi} / \varpi \\
\text { or } \sigma_{\varpi}, \text { mas }\end{array}$ & $\begin{array}{l}\sigma_{\mathrm{w}} \\
\mathrm{kpc}\end{array}$ & $\begin{array}{c}\sigma_{R_{0}}^{ \pm} \\
\mathrm{kpc}\end{array}$ & $\Delta R_{0}, \mathrm{kpc}$ \\
\hline$\sigma_{\varpi} / \varpi=0.06$ & $\begin{array}{l}0.35 \\
0\end{array}$ & $\begin{array}{l}+1.06 \\
-0.95 \\
+0.54 \\
-0.52\end{array}$ & $\begin{array}{r}-0.006_{-0.014}^{+0.015} \\
0.000_{-0.006}^{+0.007}\end{array}$ & $\sigma_{\varpi} / \varpi=0.1$ & $\begin{array}{l}0.17 \\
0\end{array}$ & $\begin{array}{l}+0.99 \\
-1.03 \\
+0.84 \\
{ }_{-0.90}\end{array}$ & $\begin{array}{l}+0.116_{-0.014}^{+0.012} \\
+0.044_{-0.012}^{+0.011}\end{array}$ \\
\hline$\sigma_{\varpi}=0.018$ & $\begin{array}{l}0.35 \\
0\end{array}$ & $\begin{array}{l}+1.55 \\
-1.39 \\
+1.23 \\
-1.16\end{array}$ & $\begin{array}{r}+0.005_{-0.019}^{+0.019} \\
+0.014_{-0.015}^{+0.018}\end{array}$ & $\sigma_{\varpi}=0.028$ & $\begin{array}{l}0.17 \\
0\end{array}$ & $\begin{array}{l}+1.71 \\
-1.49 \\
+1.65 \\
-1.45\end{array}$ & $\begin{array}{l}+0.562_{-0.019}^{+0.021} \\
+0.505_{-0.017}^{+0.023}\end{array}$ \\
\hline$\sigma_{\varpi}=0$ & 0.35 & $\begin{array}{l}+0.89 \\
-0.80\end{array}$ & $-0.012_{-0.010}^{+0.012}$ & $\sigma_{\varpi}=0$ & 0.17 & $\begin{array}{l}+0.60 \\
{ }_{-0.58}\end{array}$ & $-0.005_{-0.007}^{+0.008}$ \\
\hline
\end{tabular}

To investigate the dependence of the results on significant problem parameters, we performed series of numerical experiments in each of which one of the parameters (except $R_{0}$ and $\lambda_{0}$ ) changed in a wide neighborhood of its basic value, while other parameters retained their basic values. We will call these series basic. When varying the angular extent $\Delta \lambda$ of the segment, we fixed either its left $\left(\lambda_{1}^{\mathrm{s}}\right)$ or right edge $\left(\lambda_{2}^{\mathrm{s}}\right)$. In all cases, except the series for different $\sigma_{\varpi}$, we adopted the distance uncertainty model $\sigma_{\varpi} / \varpi=$ const, because this variant agrees better with the present-day data on masers (see Subsection 2.2). When varying the pitch angle $i, \lambda_{0}$ was chosen for each $i$ to be such that the model spiral passed through the region occupied by the masers of this arm from the data by Reid et al. (2014). The model segments for the limiting $i$ and the largest $\Delta \lambda$ are shown in Fig. 4. To clarify the role of each of the two dispersions in the problem, for some basic series we performed additional series of experiments under the assumption of $\sigma_{\varpi}=0$ or $\sigma_{\mathrm{w}}=0$. As a rule, this was done when in the basic series there were cases of biases $\Delta R_{0}$ differing significantly from zero. The results obtained are summarized in Tables 5 and 6 and are graphically presented in Figs. 5-11.

In Tables 5 and 6 the characteristics of the distribution of $R_{0, s}$ estimates are the same as those in Table 4 and are given for the extreme values of the parameters being varied for the Perseus and Scutum arm models, respectively. Figures 5-11 show the dependences of the median Me $R_{0}$ and interval [Me $R_{0}-\sigma_{R_{0}}^{-}$, Me $R_{0}+\sigma_{R_{0}}^{+}$] (gray bars) on various parameters for the $R_{0, s}$ estimates obtained in the basic and additional series for each of the two arms. In this figures the thickened bar marks the result at the basic value of the parameter being varied in a given series, while the horizontal dashed line indicates the initial $R_{0}=8 \mathrm{kpc}$.

The extent of the segment $\Delta \lambda$ turned out to be the most important parameter of the problem (Tables 5, 6 and Figs. 5, 6). The uncertainty in $R_{0}$ increases with decreasing $\Delta \lambda$, very sharply for the Perseus arm at $\Delta \lambda \lesssim 70^{\circ}$ (Fig. 5). Such short segments of an outer arm essentially impose no constraints on $R_{0}$. As the Scutum arm segment is reduced, $\sigma_{R_{0}}^{ \pm}$ increase not so dramatically, but a significant bias $\Delta R_{0}$ appears at $\Delta \lambda \lesssim 50^{\circ}$, which is attributable mainly to the nonzero spiral arm thickness, i.e., which cannot be removed by reducing the parallax errors (cf. the corresponding rows in Table 6 and the panels in Fig. 5 
Table 5. Statistical uncertainty $\left(\sigma_{R_{0}}^{+}, \sigma_{R_{0}}^{-}\right)$and mean bias $\left(\Delta R_{0}\right)$ of the $R_{0}$ estimate from the spiral-segment geometry at the extreme deviations of one of the problem parameters from its basic value for the Perseus arm models

\begin{tabular}{l||c|ll||l|ll}
\hline$p$ & $p_{\min }$ & $\sigma_{R_{0}}^{ \pm}, \mathrm{kpc}$ & $\Delta R_{0}, \mathrm{kpc}$ & $p_{\max }$ & $\sigma_{R_{0}}^{ \pm}, \mathrm{kpc}$ & \multicolumn{1}{c}{$\Delta R_{0}, \mathrm{kpc}$} \\
\hline$\Delta \lambda, \lambda_{1}^{\mathrm{s}}=-21^{\circ}$ & $50^{\circ}$ & ${ }_{-2.7}^{+5.9}$ & $-0.071_{-0.048}^{+0.045}$ & $120^{\circ}$ & ${ }_{-0.89}^{+0.94}$ & $-0.004 \pm 0.013$ \\
$\Delta \lambda, \lambda_{2}^{\mathrm{S}}=+88^{\circ}$ & $109^{\circ}$ & ${ }_{-0.94}^{+1.05}$ & $-0.006_{-0.014}^{+0.015}$ & $190^{\circ}$ & ${ }_{-0.33}^{+0.34}$ & $0.000 \pm 0.004$ \\
$\sigma_{\varpi} / \varpi$ & 0.00 & ${ }_{-0.79}^{+0.89}$ & $-0.012_{-0.010}^{+0.012}$ & 0.20 & ${ }_{-1.8}^{+2.1}$ & $-0.012_{-0.024}^{+0.034}$ \\
$\sigma_{\varpi}$, mas & 0.00 & ${ }_{-0.79}^{+0.89}$ & $-0.012_{-0.010}^{+0.012}$ & 0.05 & ${ }_{-3.1}^{+4.1}$ & $-0.020_{-0.036}^{+0.045}$ \\
$\sigma_{\mathrm{w}}, \mathrm{kpc}$ & 0.00 & ${ }_{-0.52}^{+0.54}$ & $0.000_{-0.006}^{+0.007}$ & 0.60 & ${ }_{-1.4}^{+1.7}$ & $-0.026_{-0.021}^{+0.022}$ \\
$N$ & 3 & ${ }_{-2.6}^{+3.4}$ & $-0.022_{-0.040}^{+0.030}$ & 60 & ${ }_{-0.61}^{+0.66}$ & $-0.004_{-0.009}^{+0.010}$ \\
\hline$i$ & $-20^{\circ}$ & ${ }_{-0.85}^{+0.94}$ & $+0.001_{-0.013}^{+0.008}$ & $0^{\circ}$ & ${ }_{-1.01}^{+1.14}$ & $-0.014_{-0.014}^{+0.017}$ \\
$i, \sigma_{\varpi}=0$ & $-20^{\circ}$ & ${ }_{-0.69}^{+0.77}$ & $-0.008_{-0.010}^{+0.009}$ & $0^{\circ}$ & ${ }_{-0.89}^{+1.01}$ & $-0.021_{-0.013}^{+0.015}$ \\
$i, \sigma_{\mathrm{w}}=0$ & $-20^{\circ}$ & ${ }_{-0.49}^{+0.52}$ & $-0.001_{-0.007}^{+0.006}$ & $0^{\circ}$ & ${ }_{-0.48}^{+0.49}$ & $+0.002_{-0.006}^{+0.007}$ \\
\hline
\end{tabular}

$p$ is the parameter being varied; $p_{\min }, p_{\max }$ are the minimum and maximum values of the parameter $p$. In the presence of additional conditions for our numerical experiments, they are listed in the first column.

at the basic values of the two dispersions and when either one or the other dispersion is set equal to zero). For a short segment close to the Galactic center the scatter of objects relative to the center line is not small with respect to the segment's linear extent even at accurate distances and, therefore, its curvature is established unreliably. However, at $\Delta \lambda \gtrsim 60^{\circ}$ segments close to the center can be used to estimate $R_{0}$, but only when the parallax uncertainty is necessarily taken into account in the method to avoid possible biases (the right panels in Fig. 5). At $R_{0}$ larger than the basic one the uncertainty in $R_{0}$ drops rapidly with increasing $\Delta \lambda$ for both arms, decreasing by a factor of 3 compared to the basic results (obtained at the basic values of the parameters), when the segment's extent reaches approximately half the spiral turn (Tables 5, 6 and Figs. 4, 6).

The second most important factor is the law of change in the statistical measurement error of the distance as one recedes from the Sun. Of course, as the parallax uncertainty increases, in any case, the dispersion of the $R_{0, s}$ estimates also grows, but the rate of this growth depends strongly on the model adopted for $\sigma_{\varpi}$ (Fig. 7, the panels for the basic series in Fig. 8, and Tables 5,6). Analysis of the results obtained showed that $\sigma_{R_{0}}^{ \pm}$in all of the cases considered approximately follow a power law:

$$
\sigma_{R_{0}}\left(\varsigma / \varsigma_{\mathrm{b}}\right)=k\left(\varsigma / \varsigma_{\mathrm{b}}\right)^{\alpha}+\sigma_{R_{0}}(\varsigma=0)
$$

where $\varsigma$ is a parallax dispersion characteristic $\left(\sigma_{\varpi}\right.$ or $\left.\sigma_{\varpi} / \varpi\right)$, $\varsigma_{\mathrm{b}}$ is the basic value of $\varsigma$; the index, \pm " in Eq. (5) is omitted for simplicity. The exponent $\alpha$ turned out to be virtually independent of the model for $\sigma_{\varpi}$; it is apparently specified by the segment's configuration: $\alpha \approx 1.5$ for the Perseus arm and $\alpha \approx 1$ for the Scutum arm. However, the coefficient $k$ defining the $\sigma_{R_{0}}$ growth scale for both arms in the $\sigma_{\varpi}=$ const model is several times larger $(k \approx 0.6$ for the Perseus arm and 1 for the Scutum arm) than that 
Table 6. Same as Table 5 for the Scutum arm models.

\begin{tabular}{|c|c|c|c|c|c|c|}
\hline$p$ & $p_{\min }$ & $\begin{array}{l}\sigma_{R_{0}}^{ \pm} \\
\mathrm{kpc}\end{array}$ & $\begin{array}{l}\Delta R_{0} \\
\mathrm{kpc}\end{array}$ & $p_{\max }$ & $\begin{array}{l}\sigma_{R_{0}}^{ \pm} \\
\mathrm{kpc}\end{array}$ & $\begin{array}{l}\Delta R_{0} \\
\mathrm{kpc}\end{array}$ \\
\hline$\Delta \lambda, \lambda_{1}^{\mathrm{s}}=+3^{\circ}$ & $50^{\circ}$ & $\begin{array}{l}+2.3 \\
-1.8\end{array}$ & $-0.47_{-0.03}^{+0.03}$ & $120^{\circ}$ & ${ }_{-0.93}^{+0.92}$ & $+0.294_{-0.012}^{+0.014}$ \\
\hline$\Delta \lambda, \lambda_{1}^{\mathrm{s}}=+3^{\circ}, \sigma_{\varpi}=0$ & $50^{\circ}$ & $\begin{array}{l}+2.1 \\
-1.5\end{array}$ & $-0.31_{-0.02}^{+0.02}$ & $120^{\circ}$ & $\begin{array}{l}+0.44 \\
-0.49\end{array}$ & $+0.001_{-0.005}^{+0.006}$ \\
\hline$\Delta \lambda, \lambda_{1}^{\mathrm{s}}=+3^{\circ}, \sigma_{\mathrm{w}}=0$ & $50^{\circ}$ & $\begin{array}{l}+1.1 \\
-1.2\end{array}$ & $+0.051_{-0.019}^{+0.017}$ & $120^{\circ}$ & ${ }_{-0.90}^{+0.87}$ & $+0.249_{-0.013}^{+0.010}$ \\
\hline$\Delta \lambda, \lambda_{2}^{\mathrm{s}}=+100^{\circ}$ & $97^{\circ}$ & $\begin{array}{l}+0.99 \\
-1.02\end{array}$ & $+0.11 \pm 0.01$ & $170^{\circ}$ & $\begin{array}{l}+0.31 \\
-0.29\end{array}$ & $+0.008 \pm 0.004$ \\
\hline$\Delta \lambda, \lambda_{2}^{\mathrm{s}}=+100^{\circ}, \sigma_{\varpi}=0$ & $97^{\circ}$ & ${ }_{-0.58}^{+0.60}$ & $-0.005_{-0.007}^{+0.008}$ & $170^{\circ}$ & ${ }_{-0.17}^{+0.18}$ & $-0.002_{-0.002}^{+0.003}$ \\
\hline$\Delta \lambda, \lambda_{2}^{\mathrm{s}}=+100^{\circ}, \sigma_{\mathrm{w}}=0$ & $97^{\circ}$ & $\begin{array}{l}+0.84 \\
-0.90\end{array}$ & $+0.044 \pm 0.012$ & $170^{\circ}$ & $\begin{array}{l}+0.26 \\
-0.23\end{array}$ & $+0.003_{-0.003}^{+0.004}$ \\
\hline$\sigma_{\varpi} / \varpi$ & 0.00 & $\begin{array}{l}+0.60 \\
-0.58\end{array}$ & $-0.005_{-0.007}^{+0.008}$ & 0.20 & $\begin{array}{l}+1.5 \\
-1.4\end{array}$ & $+0.48 \pm 0.02$ \\
\hline$\sigma_{\varpi} / \varpi, \sigma_{\mathrm{w}}=0$ & 0.01 & $\begin{array}{l}+0.092 \\
-0.095\end{array}$ & $0.000 \pm 0.001$ & 0.20 & $\begin{array}{l}+1.5 \\
-1.3\end{array}$ & $+0.43_{-0.02}^{+0.02}$ \\
\hline$\sigma_{\varpi}$, mas & 0.00 & $\begin{array}{l}+0.60 \\
-0.58\end{array}$ & $-0.005_{-0.007}^{+0.008}$ & 0.05 & $\begin{array}{l}+2.5 \\
-2.5\end{array}$ & $+0.69 \pm 0.03$ \\
\hline$\sigma_{\varpi}$, mas, $\sigma_{\mathrm{w}}=0$ & 0.005 & $\begin{array}{l}+0.36 \\
-0.39\end{array}$ & $-0.002_{-0.004}^{+0.005}$ & 0.05 & $\begin{array}{l}+2.3 \\
-2.4\end{array}$ & $+0.67 \pm 0.03$ \\
\hline$\sigma_{\mathrm{w}}, \mathrm{kpc}$ & 0.00 & $\begin{array}{l}+0.84 \\
-0.90\end{array}$ & $+0.044_{-0.012}^{+0.011}$ & 0.60 & $\begin{array}{l}+2.1 \\
-1.6\end{array}$ & $+0.37 \pm 0.02$ \\
\hline$\sigma_{\mathrm{w}}, \mathrm{kpc}, \sigma_{\varpi}=0$ & 0.05 & $\begin{array}{l}+0.17 \\
-0.17\end{array}$ & $-0.002 \pm 0.002$ & 0.60 & $\begin{array}{l}+2.0 \\
-1.5\end{array}$ & $+0.28 \pm 0.02$ \\
\hline$N$ & 3 & $\begin{array}{l}+2.1 \\
-1.8\end{array}$ & $+0.71_{-0.02}^{+0.02}$ & 60 & $\begin{array}{l}+0.58 \\
-0.64\end{array}$ & $-0.002_{-0.008}^{+0.007}$ \\
\hline$N, \sigma_{\varpi}=0$ & 3 & $\begin{array}{l}+1.5 \\
-1.2\end{array}$ & $+0.17 \pm 0.02$ & 60 & $\begin{array}{l}+0.33 \\
-0.32\end{array}$ & $-0.004 \pm 0.004$ \\
\hline$N, \sigma_{\mathrm{w}}=0$ & 3 & $\begin{array}{l}+1.7 \\
-1.5\end{array}$ & $+0.58 \pm 0.03$ & 60 & $\begin{array}{l}-0.54 \\
+0.49\end{array}$ & $-0.007 \pm 0.006$ \\
\hline$i$ & $-20^{\circ}$ & $\begin{array}{l}+0.99 \\
-1.03\end{array}$ & $+0.11 \pm 0.01$ & $0^{\circ}$ & $\begin{array}{l}+1.5 \\
-1.5\end{array}$ & $+0.33 \pm 0.02$ \\
\hline$i, \sigma_{\varpi}=0$ & $-20^{\circ}$ & $\begin{array}{l}+0.60 \\
-0.58\end{array}$ & $-0.005 \pm 0.01$ & $0^{\circ}$ & $\begin{array}{l}+0.86 \\
-0.88\end{array}$ & $+0.064_{-0.015}^{+0.013}$ \\
\hline$i, \sigma_{\mathrm{w}}=0$ & $-20^{\circ}$ & $\begin{array}{l}+0.84 \\
-0.90\end{array}$ & $+0.044_{-0.012}^{+0.012}$ & $0^{\circ}$ & $\begin{array}{l}+1.4 \\
-1.3\end{array}$ & $+0.22 \pm 0.02$ \\
\hline
\end{tabular}

for $\sigma_{\varpi} / \varpi=$ const $(k \approx 0.2$ and 0.4 , respectively). As a result, for example, the ratio $\sigma_{R_{0}}(2) / \sigma_{R_{0}}(0)$ is $\sim 1.5$ for the Perseus arm and $\sim 2.5$ for the Scutum arm at $\sigma_{\varpi} / \varpi=$ const, while at $\sigma_{\varpi}=$ const it is $\sim 3$ and $\sim 4.5$, respectively. On the other hand, the complete absence of errors in the parallaxes increases the statistical accuracy of the $R_{0, s}$ estimate compared to the basic result at $\sigma_{\varpi}=$ const much more dramatically (almost by a factor of 3 for the Scutum arm) than it does at $\sigma_{\varpi} / \varpi=$ const (see Table 4 ).

The dispersion of the $R_{0, s}$ estimates expectedly decreases with increasing number $N$ of objects representing the segment; in the case of the Perseus arm, and for $N / 3 \geqslant 8$ in the case of the Scutum arm, the agreement with the law

$$
\sigma_{R_{0}}^{ \pm}(N) \propto N^{-1 / 2}
$$

is almost perfect (Fig. 9, upper panels). Poorer agreement for smaller $N$ in the case of the second arm is obtained in the presence of a significant bias $\Delta R_{0}$. The latter is caused mainly by the errors in the parallaxes (cf. the two lower and upper right panels in Fig. 9). At $\sigma_{\varpi}=0$ mas close agreement with the law (6) is obtained already at $N / 3 \geqslant 3$ (the lower left panel in Fig. 9). The drop in $\sigma_{R_{0}}^{ \pm}(N)$ according to the law (6) and the absence of significant biases $\Delta R_{0}$ at least at $N \geqslant 24$ (and for the Perseus arm also at small $N$ ) 

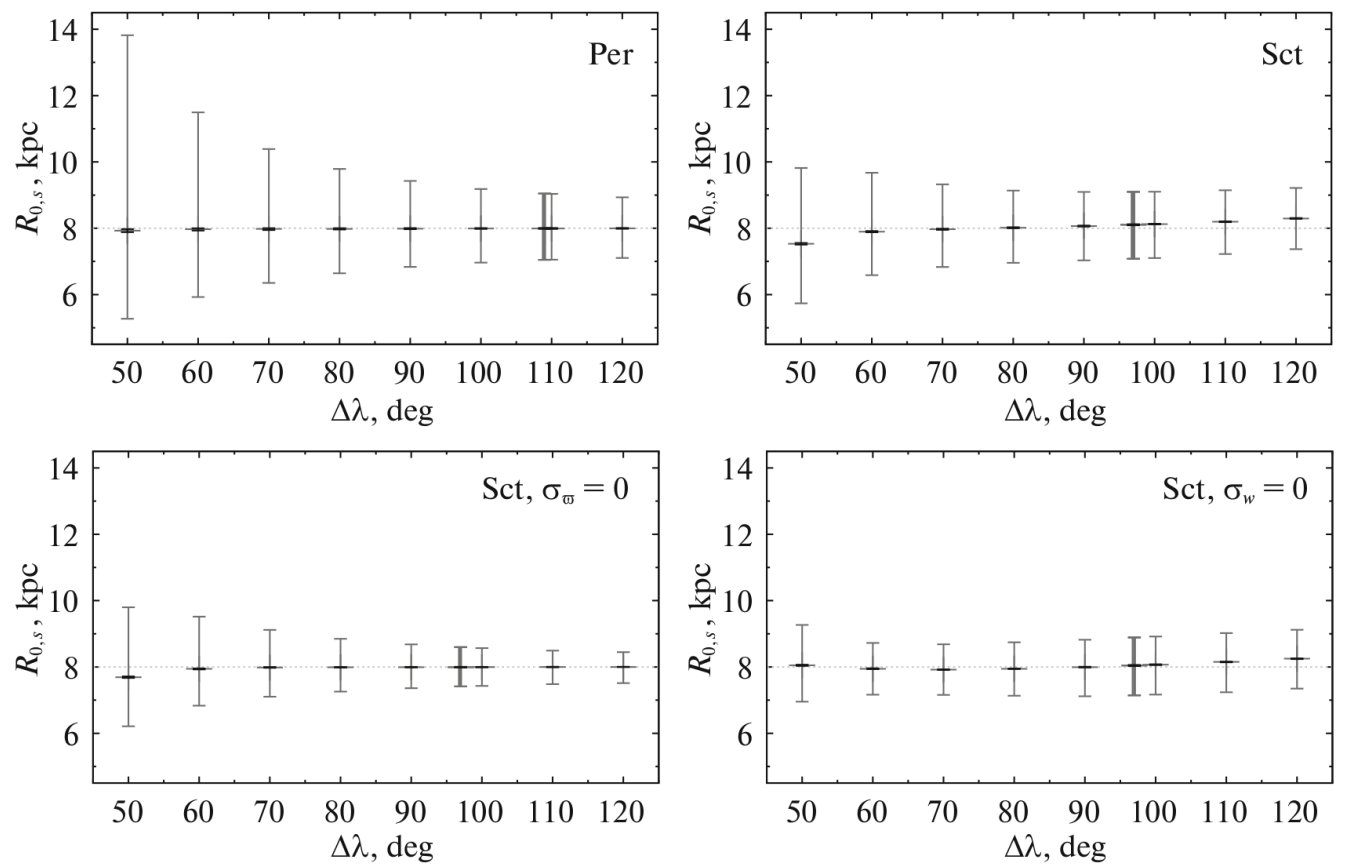

Fig. 5. Median and statistical uncertainty (the $1 \sigma$ interval $\left[\mathrm{Me} R_{0}-\sigma_{R_{0}}^{-}\right.$, Me $R_{0}+\sigma_{R_{0}}^{+}$, gray bars) of the $R_{0}$ estimates from the spiral-segment geometry as a function of the segment's angular extent in the case of a constant longitude of its left edge $\left(\lambda_{1}^{\mathrm{s}}\right)$ for the Perseus and Scutum arm models. On each panel the thickened gray bar marks the result at the basic value of the parameter being varied. The small black bars indicate the $1 \sigma$ uncertainty of the median $\mathrm{Me} R_{0}$.

provide evidence for the consistency of the $R_{0}$ estimate from the spiral-segment geometry even in the case of applying a simplified (three-point) method (when $N \rightarrow \infty$ the mean $\mathrm{M} R_{0, s} \rightarrow R_{0}$ and the dispersion $\left.\mathrm{D} R_{0, s} \rightarrow 0\right)$.

The values of $\sigma_{R_{0}}^{ \pm}$grow with increasing $\sigma_{\mathrm{w}}$, along with increasing $\sigma_{\varpi}$ and $\sigma_{\varpi} / \varpi$, approximately according to a power law (Fig. 10). The role of the $\sigma_{\mathrm{w}}$ contribution to the total uncertainty of the $R_{0, s}$ estimate depends on the parallax accuracy level and the form of the dependence $\sigma_{\varpi}(r)$. For example, for the Perseus arm, when the scatter $\sigma_{\mathrm{w}}$ is set equal to zero, $\sigma_{R_{0}}^{ \pm}$decrease by half for $\sigma_{\varpi} / \varpi=$ const and only by $\approx 1 / 4$ for $\sigma_{\varpi}=$ const (Table 4, the "Per" panel in Fig. 10). For the Scutum arm the contribution of the parallax errors dominates in both models for $\sigma_{\varpi}$. Therefore, setting $\sigma_{\mathrm{w}}$ equal to zero in both cases reduces $\sigma_{R_{0}}^{ \pm}$insignificantly at the basic values of $\sigma_{\varpi}$ and $\sigma_{\varpi} / \varpi$ (Table 4 , the "Sct" panels in Fig. 10).

The dispersion of the $R_{0, s}$ estimate is least affected by the pitch angle $i$ of the model spiral (Fig. 11 and Tables 5, 6). At the basic values of both dispersions in the problem the general trend with changing $i$ is not quite obvious (the upper panels in Fig. 11). However, setting $\sigma_{\varpi}$ equal to zero gives a clear picture: $\sigma_{R_{0}}^{ \pm}$grow almost linearly with increasing $|i|$ (the middle panels in Fig. 11). This means that in the absence of measurement errors the distance from the Sun to the center of the ring structure is determined from its sector with a lower (!) accuracy than the distance to the pole of the spiral arm from its segment at the same angular extent of the sector and the segment. The larger the arm pitch angle in absolute value, the more accurate the result. The growth of $\sigma_{R_{0}}^{ \pm}$ with $|i|$ is moderate, but not negligible: at $\sigma_{\varpi}=0$ mas in the interval $i \in\left[-20^{\circ}, 0^{\circ}\right]$ it is 

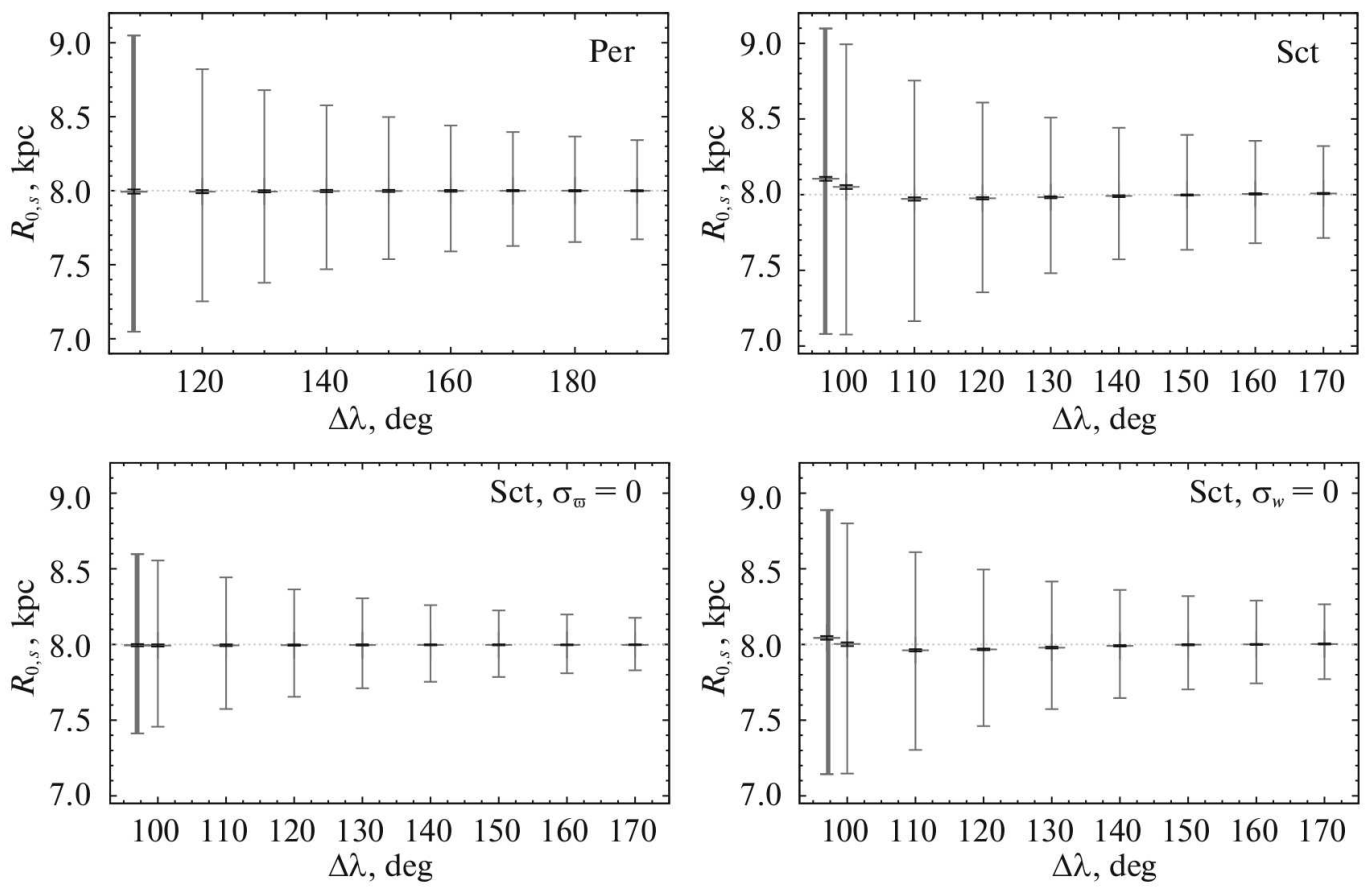

Fig. 6. Same as Fig. 5 in the case of a constant longitude of the segment's right edge $\left(\lambda_{2}^{\mathrm{s}}\right)$.
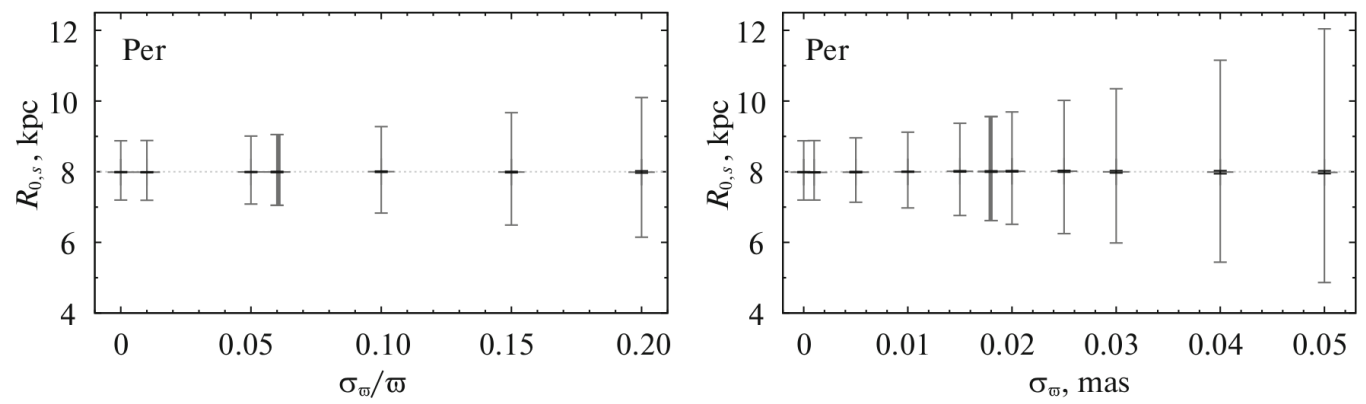

Fig. 7. Same as Fig. 5, but as a function of relative and absolute rms parallax errors for the Perseus arm models.

a factor of $\approx 1.3$ for the Perseus arm and a factor of $\approx 1.5$ for the Scutum arm (Tables 5 and 6$)$. For ideally thin structures $\left(\sigma_{\mathrm{w}}=0 \mathrm{kpc}\right)$ distorted by the errors in the parallaxes, the behavior of $\sigma_{R_{0}}^{ \pm}(i)$ is determined by the scatter and characteristic values of distances to the points of the segment from the Sun and from the Galactic center, the position of the segment relative to the center-anticenter axis, and other parameters. Therefore, for different configurations the trends can be quite different (the lower panels in Fig. 11), whence the complex dependences $\sigma_{R_{0}}^{ \pm}(i)$ under the combined action of both dispersions (the upper panels in Fig. 11).

\section{DISCUSSION}

Significant biases $\Delta R_{0}$ were detected only for the Scutum arm (Table 6, Figs. 5, 6, 8-11). This stems from the fact that its segments have an appreciably smaller linear extent and are much closer to the Galactic center than the Perseus arm segments (Fig. 4). As a result, the solutions for the Scutum arm turn out to be more sensitive to the dispersion relative to the center line. In this case, the errors in the parallaxes play a major role in the 

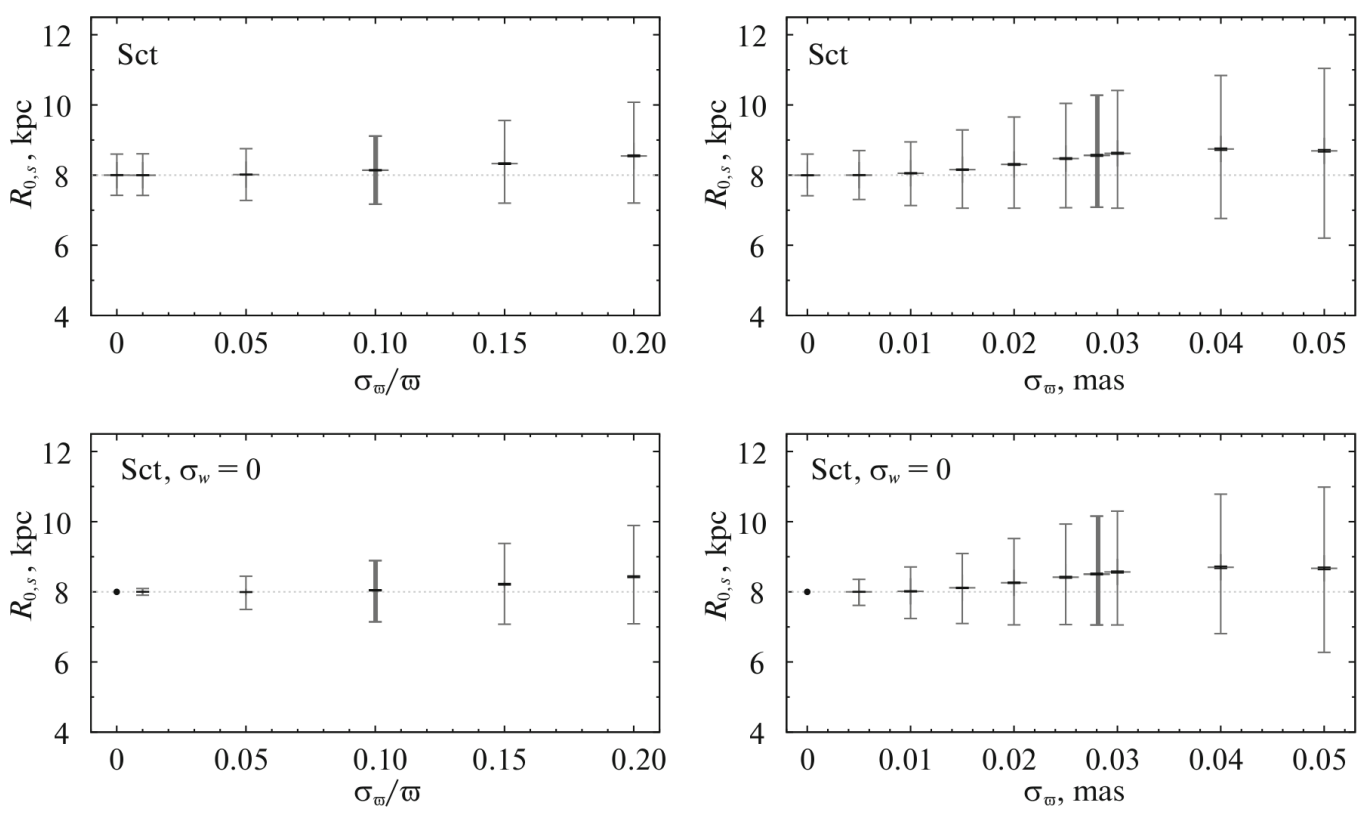

Fig. 8. Same as Fig. 7 for the Scutum arm models.
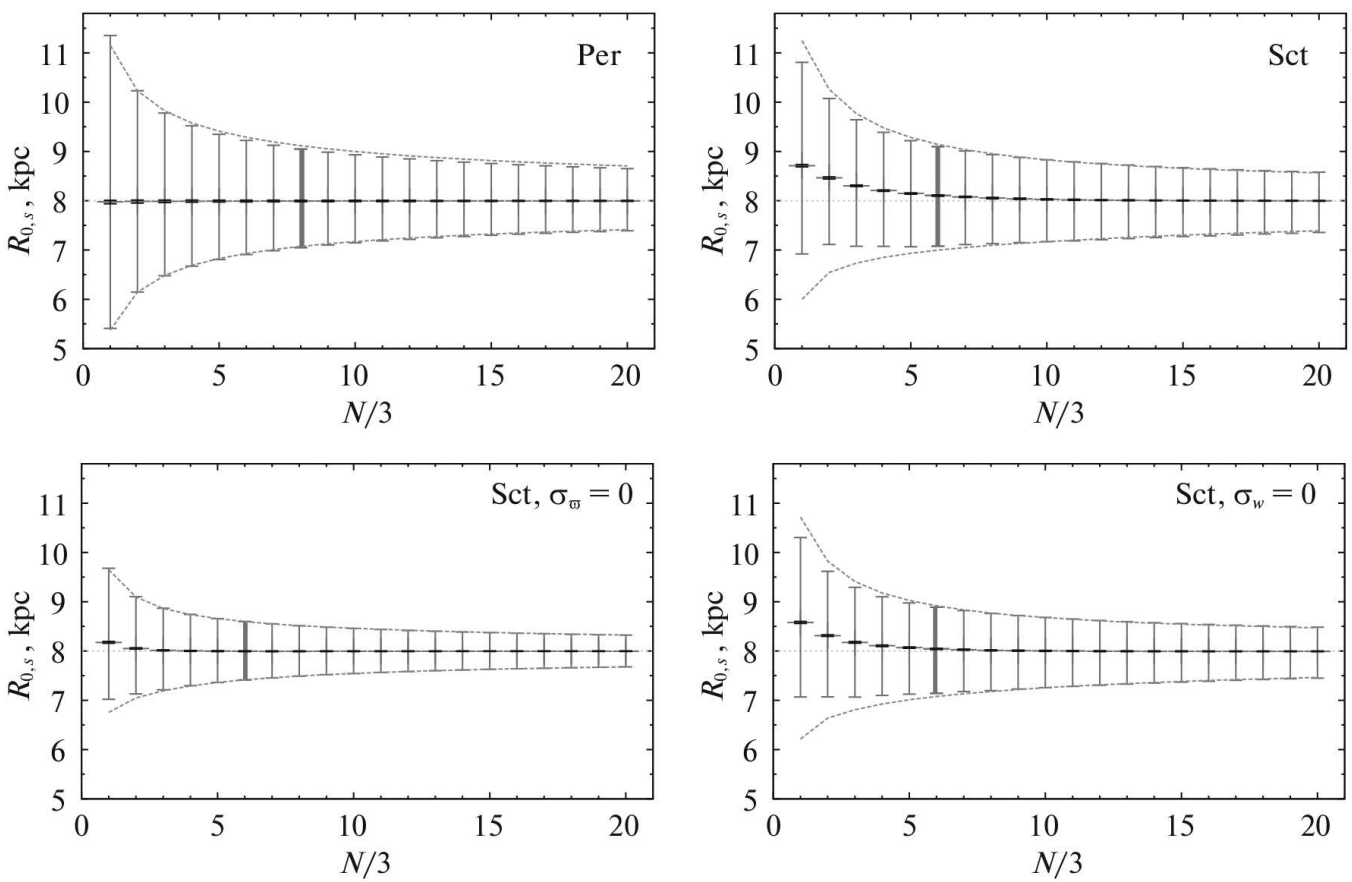

Fig. 9. Same as Fig. 5, but as a function of the number of objects populating the segment. The curves represent the laws $\sigma_{R_{0}}^{+}(N) \propto 1 / \sqrt{N}$ and $\sigma_{R_{0}}^{-}(N) \propto 1 / \sqrt{N}$.

appearance of significant biases: when they are set equal to zero, these biases disappear in most cases (cf. the results of the basic series and additional series at $\sigma_{\varpi}=0$ in the table and the figures mentioned above). The biases $\Delta R_{0}$ caused by the parallax uncertainty are always positive (see the results at $\sigma_{\mathrm{w}}=0$ there). For a normal distribution of random parallax errors the distribution of distance errors has a positive skewness, leading to some overestimation of the distance, on average, which is greater for larger $\sigma_{\varpi}$. In combination with the location of the Scutum arm in the general direction to the Galactic center, this leads to an overestimation of $R_{0, s}$ from the segments of this arm, on average. However, 

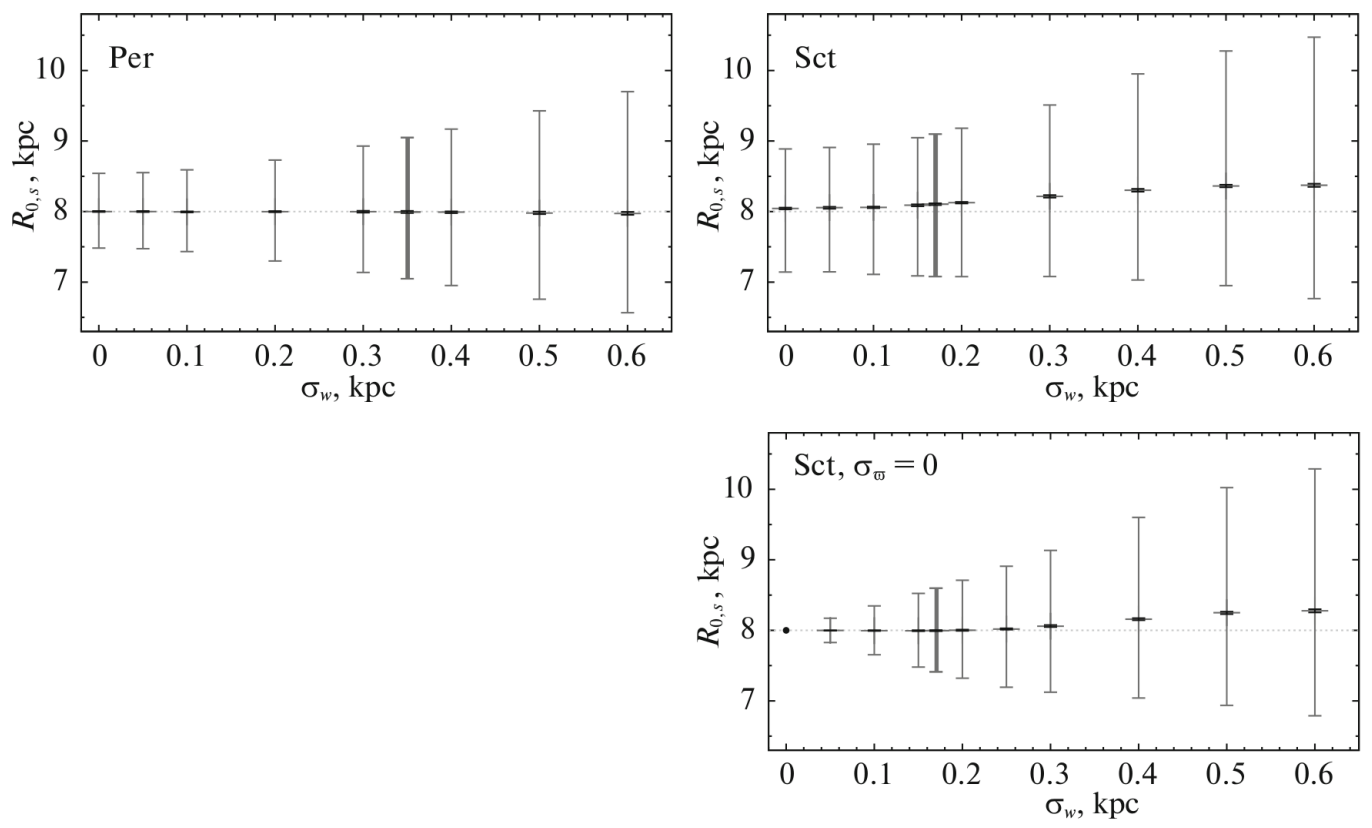

Fig. 10. Same as Fig. 5, but as a function of the scatter of objects across the model spiral segment.

the biases are not always explained only by the errors in the parallaxes: at a small extent $\left(\Delta \lambda \lesssim 60^{\circ}\right)$, a sparse population $(N / 3 \leqslant 2)$, and a large natural scatter $\left(\sigma_{\mathrm{w}} \gtrsim 0.3 \mathrm{kpc}\right)$ small, but significant biases $\Delta R_{0}=-0.3 \div+0.3 \mathrm{kpc}$ can result even for $\sigma_{\varpi}=0$ mas (Table 6; the "Sct, $\sigma_{\varpi}=0$ " panels, Figs. 5, 9, 10). Their nature is different: if the dispersion is not small compared to the segment length, then for a significant fraction of the sets of triplets $M_{j}^{\prime \prime}$ (for example, lying on one straight line) the information about the curvature of the initial segment is completely lost; the formal solutions of (2) for such triplets lead to biases. To avoid noticeable biases $\Delta R_{0}$, we need: (1) to take into account the errors in the parallaxes directly in the method and (2) not to use short, sparsely populated, disperse segments close to the Galactic center for our spatial modeling.

Note that in our preliminary numerical experiments (Nikiforov and Veselova 2015) significant biases were also obtained for the Perseus arm in some series. This was caused by the exclusion of the triplets of points $M_{j}^{\prime \prime}$ corresponding to leading spirals $\left(i>0^{\circ}\right)$ from consideration. Our new experiments showed that, despite the seeming naturalness of this constraint for our Galaxy, it actually cuts off part of the distribution of $R_{0, s}$ estimates and, thus, can introduce a fictitious bias $\Delta R_{0}$. When abandoning the constraint $i \leqslant 0^{\circ}$, the biases for the Perseus arm disappear. In this paper we did not impose this constraint.

In all series of experiments for the Perseus arm and in most of the series for the Scutum arm it turned out that $\sigma_{R_{0}}^{+}>\sigma_{R_{0}}^{-}$(see the tables and figures in Section 3). This is partly a consequence of the skewness of the distribution of distance errors discussed above and partly a general geometric property of some classes of methods for determining $R_{0}$, i.e., the data agree better with the model at the trial $R_{0}^{\prime}=\breve{R}_{0}+\delta R_{0}$ than at $R_{0}^{\prime}=\breve{R}_{0}-\delta R_{0}$, where $\breve{R}_{0}$ is the optimal or true value, especially if $\delta R_{0}>0 \mathrm{kpc}$ is not small compared to $\breve{R}_{0}$ (see Nikiforov $(1999,2000)$ and Nikiforov and Kazakevich (2009) as examples in the kinematic class of methods). 

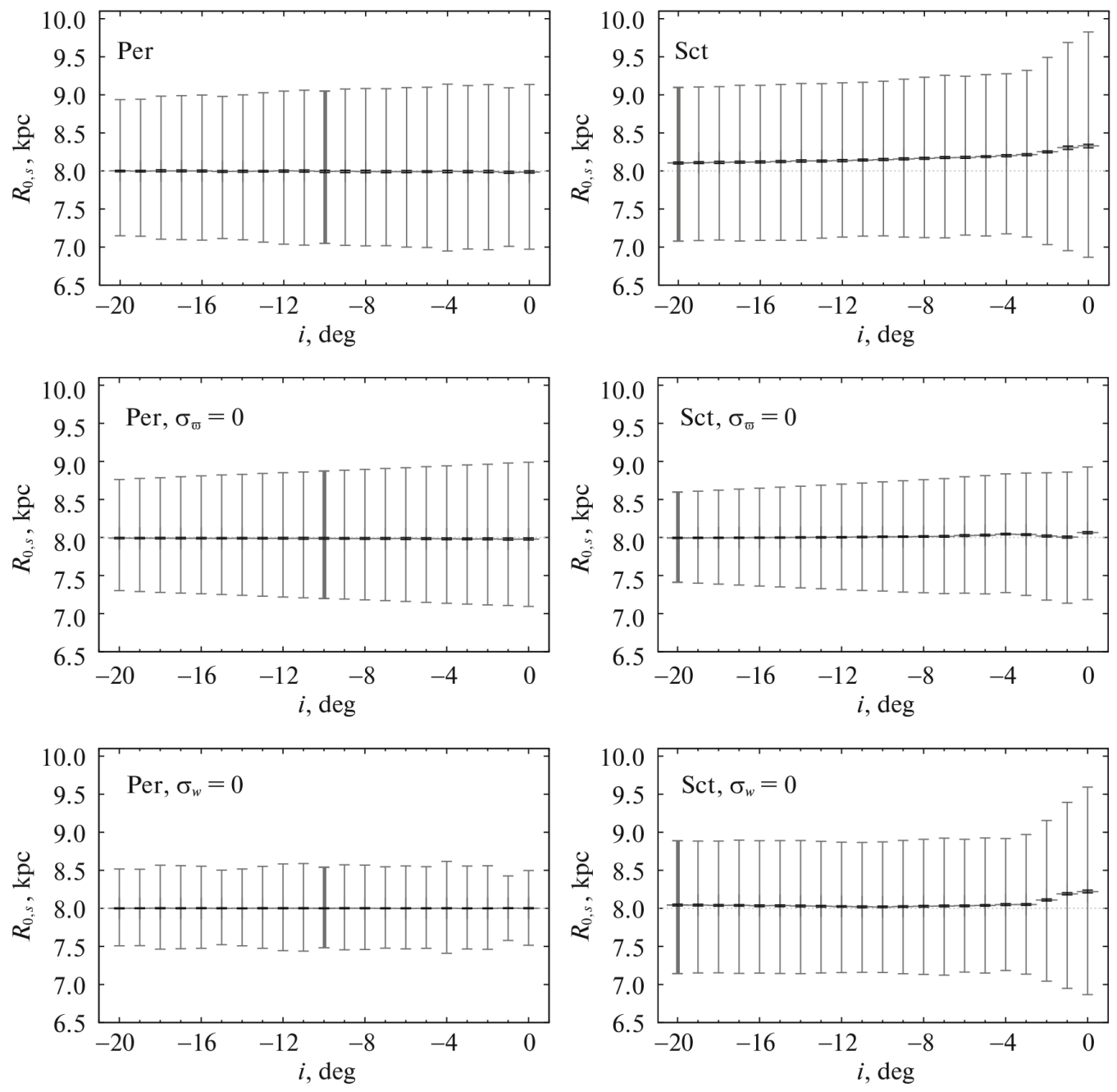

Fig. 11. Same as Fig. 5, but as a function of model spiral pitch angle.

The results obtained in the previous section show that the dispersion of the $R_{0}$ measurements from the geometry of spiral segments depends significantly on the law $\sigma_{\varpi}(r)$. As an example, we generated pseudorandom "observed" distributions of objects (100 per each degree of longitude $\lambda$ ) belonging to one turn of a spiral arm with parameters equal to the basic values for the Perseus arm at a constant absolute parallax uncertainty (Fig. 12a) and a constant relative parallax uncertainty (Fig. 12b). These figures illustrate how strongly the true distribution of spiral arm tracers for the $\sigma_{\varpi}=$ const model is smeared compared to the $\sigma_{\varpi} / \varpi=$ const model. The fact that the present-day data on masers are closer to the latter model (Subsection 2.2) is a very lucky circumstance for the prospects of applying this approach. In any case, the errors in the parallaxes deform noticeably the observed spatial distribution and, therefore, its proper modeling is possible only when both dispersions are taken into account. In some spiral arm segments a particular dispersion dominates, while in other segments the dispersions are comparable in importance (Figs. 12b and 12c). The results of our experiments in Section 3 also indicate that, although, on the whole, the distance measurement errors are a more important factor, the contributions of the two dispersions to the total uncertainty in the $R_{0, s}$ estimate are comparable (cf. the results at $\sigma_{\varpi}=0$ mas and $\sigma_{\mathrm{w}}=0 \mathrm{kpc}$ ). This implies that none of the dispersions can be ignored in 

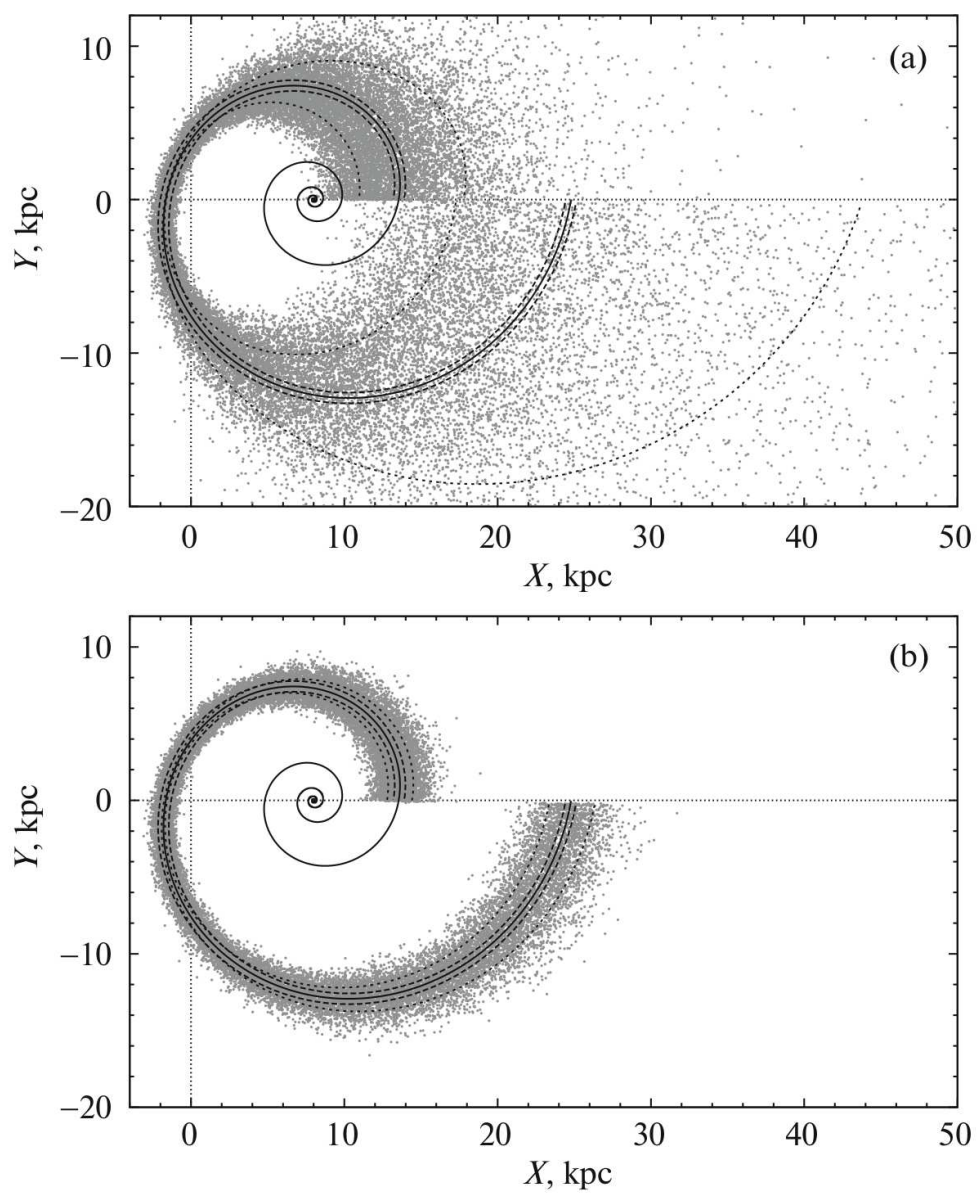

Fig. 12. Numerically generated distribution of 3600 objects belonging to one turn of a spiral arm with parameters of its center line $R_{0}=8.0 \mathrm{kpc}, i=-10^{\circ}, \lambda_{0}=61^{\circ}$ and a transverse scatter $\sigma_{\mathrm{w}}=0.35 \mathrm{kpc}$ at $\sigma_{\varpi}=0.0175$ mas (a) and $\sigma_{\varpi} / \varpi=0.06(\mathrm{~b})$. The solid line indicates the model spiral, the dashed and dotted lines bound the region of deviation from the model spiral by $\pm 1 \sigma_{\mathrm{w}}$, and by $\pm 1 \sigma_{\varpi}$ (a) or $\pm 1 \sigma_{\varpi} / \varpi(\mathrm{b})$, respectively.

our modeling.

For both arms the statistical uncertainty is $\sigma_{R_{0}} \approx 1 \mathrm{kpc}$ at the basic segment parameters (Table 4 ). As has been shown in the previous section, the dispersion of the $R_{0, s}$ estimates is most strongly affected by the extent $\Delta \lambda$ of the segment. Therefore, it can be assumed that the presence of edge objects, which, in reality, specify the segment boundaries, must slightly increase the conditionality of the $R_{0}$ determination problem compared to the results of modeling the segment by three representative points. Thus, one might expect the $\sigma_{R_{0}}$ estimates obtained in our experiments to be upper limits. To estimate the overestimation coefficient, we repeated the numerical experiments in NV18 for masers distributed over the segment (when abandoning the constraint on $i$ ) and found $\sigma_{R_{0}}^{-}=0.84 \mathrm{kpc}$, $\sigma_{R_{0}}^{+}=0.85 \mathrm{kpc}$ for the Perseus arm and $\sigma_{R_{0}}^{-}=0.73 \mathrm{kpc}, \sigma_{R_{0}}^{+}=0.65 \mathrm{kpc}$ for the Scutum arm. Taking the largest of these quantities and comparing it with the basic results in this paper, we find that the overestimation of $\sigma_{R_{0}}^{ \pm}$in our three-point experiments is at least $15 \%$ at parameters close to the basic ones, i.e., as the uncertainty from only one segment we may take a cautious estimate $\sigma_{R_{0}} \simeq 0.85 \mathrm{kpc}$. If the data on several $\left(N_{\mathrm{arm}}\right)$ segments 
are used, then, other things being equal, one might expect the final uncertainty

$$
\sigma_{R_{0}} \simeq \frac{0.85 \mathrm{kpc}}{\sqrt{N}_{\mathrm{arm}}}
$$

For example, $\sigma_{R_{0}} \simeq 0.5 \mathrm{kpc}$ for three segments (open clusters), $\sigma_{R_{0}} \simeq 0.4 \mathrm{kpc}$ for four segments (masers), and $\sigma_{R_{0}} \simeq 0.3 \mathrm{kpc}$ for eight segments (classical Cepheids). This is close to the statistical accuracy of present-day $R_{0}$ estimates (see, e.g., Bland-Hawthorn and Gerhard 2016; de Grijs and Bono 2016).

Using short and sparsely populated segments as well as intrinsically less accurate data on the heliocentric distances can reduce the predicted accuracy of the solution. If the law $\sigma_{\varpi}=$ const is established in future when measuring the parallaxes, then the question about $\sigma_{\varpi}$ will become acute. If, however, the law $\sigma_{\varpi} / \varpi=$ const is retained, then the relative parallax accuracy will be not so important.

The accuracy of $R_{0}$ can be improved most dramatically by increasing the extent of the identified segments. The next most important factors for refining the solution are an increase in the number of objects with independently measured distances attributed to the segments and revealing new arms segments and the internal structure of already known features in the spiral pattern. The potential for reducing $\sigma_{R_{0}}$ through a more accurate parallax measurement (at $\sigma_{\varpi} / \varpi=$ const) is only moderate.

The presented results were obtained with a standard multiplicative congruent random number generator (IBM Manual C20-8011), which has an acceptable quality for our purposes in the two-dimensional case. To check the stability of our results to the choice of a generator, we also used one of the generators by Fishman and Moore (1986), the first in their rating belonging to the same class for the most dispersive series of experiments. With this generator for the Perseus arm the biases $\Delta R_{0}$ differ from those presented in the paper within the error limits, while the standards $\sigma_{R_{0}}^{ \pm}$retain all of the presented signs. For the Scutum arm with these two generators the standards differ insignificantly within the entire series, while the biases differ at moderate deviations of the parameter being varied from the basic value. For the extreme values of the parameter, at which the solution for the Scutum arm is unstable for the above reasons, with the second generator the biases turn out to be slightly smaller, but remain significant in all cases when they are significant with the first generator. Thus, the application of a different random number generator does not affect our conclusions.

On the whole, our results show that the geometric estimation of $R_{0}$ from spiral segments is operable for a wide set of possible parameters even in the case of a simplified (three-point method). However, the capabilities of this, basically test method are limited: it disregards the natural and measuring dispersions; it is too sensitive to some peculiarities of the spatial distribution of segment objects, especially at a small sample size (see NV18); the solution can be directly obtained with its help only for one segment and for one type of model (logarithmic spiral). As was pointed out in NV18, both dispersions can be taken into account when implementing this approach within the maximum likelihood method (MLM). Although this method will be much more laborious, it will allow one to estimate 
$R_{0}$ from the geometry of spiral segments for arbitrary arm models and, simultaneously, to properly solve classical and new problems of modeling the Galactic spiral structure.

Note that the possibility of introducing a more general arm model is important for eliminating the external error within this approach. In the case of the three-point method, systematic deviations from a logarithmic spiral can lead to such an error. For example, if the representative points are taken from an Archimedean spiral, then for the basic segments of the Perseus and Scutum arms the three-point method gives the biases $\Delta R_{0}=$ -0.02 and +0.06 kpc, respectively. However, according to our preliminary estimates, significant deviations from the shape of a logarithmic spiral cannot yet be found from tracers with reliable photometric or absolute distances for the arms of our Galaxy. Among the various cases, the largest (in absolute value) derivative of the pitch angle $i_{1} \equiv \frac{d i}{d \lambda}$ is $+0.029 \pm 0.076$ for the Perseus arm and $-0.04 \pm 0.25$ for the Scutum arm 1 (both estimates differ insignificantly from zero). If the representative points are taken from spirals with a variable pitch angle $i(\lambda)=i_{0}+i_{1}\left(\lambda-\lambda_{0}\right)$, then taking these values of $i_{1}$, this gives upper (in absolute value) limits for the biases in the three-point method: $\Delta R_{0}<+0.6 \mathrm{kpc}$ for the Perseus arm and $\Delta R_{0}>-0.4 \mathrm{kpc}$ for the Scutum arm. In all these cases, the nonlogarithmic spirals in the segment between the representative points at the basic segment extent differ little from the logarithmic models compared to the uncertainty of the latter, while the biases for individual segments are smaller or even appreciably smaller than the statistical errors in $R_{0}$ and have opposite signs for different segments. Therefore, one might expect the bias of the final $R_{0}$ estimate from several segments to be insignificant. In any case, a bias of this type is not a fundamental problem of our method for determining $R_{0}$ provided that we pass to the MLM that admits using more general models. This will allow one to reveal systematic deviations from a logarithmic spiral, if they exist, and, simultaneously, to take them into account when estimating the parameters. On the other hand, the scale of potential biases for individual segments shows that attention should be paid to searching for such deviations.

\section{CONCLUSIONS}

To establish the capabilities and applicability boundaries of the new method for determining the distance to the Galactic center $R_{0}$ proposed by us previously (NV18), from the geometry of spiral arm segments, we investigated the influence of various factors on the statistical properties of the $R_{0}$ estimate from a separate segment through numerical simulations. The $R_{0}$ estimates were found by a simplified method reconstructing the geometry of a segment from its three representative points, which allowed a large number of numerical experiments to be performed in a limited time. The problem parameters were varied in wide neighborhoods of their basic values characterizing the Perseus and Scutum arm segments from the present-day data on masers with trigonometric parallaxes.

The statistical uncertainty $\sigma_{\varpi}$ in the present-day parallax measurements for masers was shown to systematically decrease (!) with increasing heliocentric distance $r$, with

\footnotetext{
${ }^{1}$ Here $i$ and $\lambda$ are expressed in the same angular measure.
} 
the relative uncertainty $\sigma_{\varpi} / \varpi$ remaining, on average, approximately constant (at least at $r \lesssim 3.2 \mathrm{kpc})$.

Our numerical experiments provide evidence for the consistency of the $R_{0}$ estimate from the spiral-arm geometry. Significant biases of the estimate were detected only for the inner arm (Scutum); they are attributable mainly to the random errors in the parallaxes, which lead to an asymmetric distribution of distances $r$, as well as the small angular extent $\Delta \lambda$ of the segment and the small number of $N$ of objects representing it. The dispersion of the $R_{0}$ estimate is affected most strongly by the extent $\Delta \lambda$ (as the latter increases from the basic value to half the spiral turn, $\sigma_{R_{0}}$ decreases by a factor of 3 ). As the parallax uncertainty grows, $\sigma_{R_{0}}$ increases. If in further parallax measurements $\sigma_{\varpi}$, on average, remains constant with $r$, then $\sigma_{\varpi}$ will be almost equally important for the dispersion of $R_{0}$ as $\Delta \lambda$. When the law $\sigma_{\varpi} / \varpi=$ const, which describes well the presentday data, is retained, the remaining parameters, except the pitch angle $i$, exert an equally significant, but weaker influence on $\sigma_{R_{0}}$. In the absence of parallax errors a decrease in $|i|$ increases little the dispersion of $R_{0}$; in particular, this implies that when the spiral segment degenerates into a ring sector $\left(\right.$ at $i=0^{\circ}$ ), the distance to the center of the latter is determined less accurately than the distance to the pole of the spiral segment.

The applicability boundaries of the $R_{0}$ determination from the spiral-segment geometry are limited by the dispersion of the $R_{0}$ estimate $\left(\Delta \lambda>70^{\circ}, \sigma_{\varpi}<0.04\right.$ mas, $N / 3>1$ ) for the outer arm (Perseus) and by the presence of a significant bias in the $R_{0}$ estimate caused not by the parallax errors, but by the finite segment thickness $\left(\Delta \lambda>50^{\circ}\right.$, $\sigma_{\mathrm{w}}<0.3 \mathrm{kpc}, N / 3>2$ ) for the inner arm (Scutum).

The fact that the present-day data on masers agree better with the $\sigma_{\varpi} / \varpi=$ const model is a very lucky circumstance for applying our approach to these tracers of the spiral structure. The accuracy of the final $R_{0}$ estimate can be improved by using several arm segments in the analysis and by increasing the extent $\Delta \lambda$ of the identified segments and the number of objects with independent distance estimates attributed to them.

Our results suggest that the $R_{0}$ estimation from spiral segments is operable for a wide set of possible parameters even when using a robust, but inefficient L-estimator (median) in the three-point method. This makes the development of a more complex, but more correct method based on an efficient M-estimator meaningful. As our numerical experiments showed, the combined action of the measuring dispersion of distances and the natural scatter across the arm generally leads to a more complex form of the observed spatial distribution of objects, with the influence of one of the dispersions on it, on the whole, being comparable to the influence of the other one. To properly model this distribution, the maximum-likelihood (ML) estimators can be used as an M-estimator.

\section{ACKNOWLEDGMENTS}

We are grateful to the referees for their useful remarks. This work was supported by the Russian Science Foundation (grant no. 18-12-00050). 


\section{REFERENCES}

1. J. Bland-Hawthorn and O. Gerhard, Ann. Rev. Astron. Astrophys. 54, 529 (2016).

2. T. Camarillo, V. Mathur, T. Mitchell, and B. Ratra, Publ. Astron. Soc. Pacif. 130, 024101 (2018).

3. X. Chen, S. Wang, L. Deng, and R. de Grijs, Astrophys. J. 859, 137 (2018).

4. A. K. Dambis, L. N. Berdnikov, Yu. N. Efremov, A. Yu. Kniazev, A. S. Rastorguev, E. V. Glushkova, V. V. Kravtsov, D. G. Turner, et al., Astron. Lett. 41, 489 (2015).

5. G. S. Fishman and L. R. Moore, SIAM J. Sci. Stat. Comput. 7, 24 (1986).

6. R. de Grijs and G. Bono, Astrophys. J. Suppl. Ser. 227, 5 (2016).

7. E. Griv, I.-G. Jiang, and L.-G. Hou, Astrophys. J. 844, 118 (2017).

8. A. I. Kobzar', Applied Mathematical Statistics. For Engineers and Scientists (Fizmatlit, Moscow, 2006) [in Russian].

9. V. Krishnan, S. P. Ellingsen, M. J. Reid, H. E. Bignall, J. McCallum, C. J. Phillips, C. Reynolds, and J. Stevens, Mon. Not. R. Astron. Soc. 465, 1095 (2017).

10. D. Majaess, I. Dekany, G. Hajdu, D. Minniti, D. Turner, and W. Gieren, Astrophys. Space Sci. 363, 127 (2018).

11. F. Mignard, Astron. Astrophys. 354, 522 (2000).

12. I. I. Nikiforov, Astron. Rep. 43, 345 (1999).

13. I. I. Nikiforov, ASP Conf. Ser. 209, 403 (2000).

14. I. I. Nikiforov, ASP Conf. Ser. 316, 199 (2004).

15. I. I. Nikiforov and E. E. Kazakevich, Izv. GAO 219 (4), 245 (2009).

16. I. I. Nikiforov and O. V. Smirnova, Astron. Nachr. 334, 749 (2013).

17. I. I. Nikiforov and A. V. Veselova, Baltic Astron. 24, 387 (2015).

18. I. I. Nikiforov and A. V. Veselova, Astron. Lett. 44, 81 (2018).

19. M. E. Popova and A. V. Loktin, Astron. Lett. 31, 171 (2005).

20. W. H. Press, S. A. Teukolsky, W. T. Vetterling, and B. P. Flannery, Numerical Recipes in C (Cambridge Univ. Press, Cambridge, UK, 1997).

21. A. S. Rastorguev, N. D. Utkin, M. V. Zabolotskikh, A. K. Dambis, A. T. Bajkova, and V. V. Bobylev, Astrophys. Bull. 72, 122 (2017).

22. M. J. Reid, private commun. (2014). 
23. M. J. Reid, K. M. Menten, A. Brunthaler, X. W. Zheng, T. M. Dame, Y. Xu, Y. Wu, B. Zhang, et al., Astrophys. J. 783, 130 (2014). 